\title{
Common zero sets of equivalent singular inner functions
}

\author{
by \\ KeIJI Izuchi (Niigata)
}

\begin{abstract}
Let $\mu$ and $\lambda$ be bounded positive singular measures on the unit circle such that $\mu \perp \lambda$. It is proved that there exist positive measures $\mu_{0}$ and $\lambda_{0}$ such that $\mu_{0} \sim \mu$, $\lambda_{0} \sim \lambda$, and $\left\{\left|\psi_{\mu_{0}}\right|<1\right\} \cap\left\{\left|\psi_{\lambda_{0}}\right|<1\right\}=\emptyset$, where $\psi_{\mu}$ is the associated singular inner function of $\mu$. Let $\mathcal{Z}(\mu)=\bigcap_{\{\nu ; \nu \sim \mu\}} Z\left(\psi_{\nu}\right)$ be the common zeros of equivalent singular inner functions of $\psi_{\mu}$. Then $\mathcal{Z}(\mu) \neq \emptyset$ and $\mathcal{Z}(\mu) \cap \mathcal{Z}(\lambda)=\emptyset$. It follows that $\mu \ll \lambda$ if and only if $\mathcal{Z}(\mu) \subset \mathcal{Z}(\lambda)$. Hence $\mathcal{Z}(\mu)$ is the set in the maximal ideal space of $H^{\infty}$ which relates naturally to the set of measures equivalent to $\mu$. Some basic properties of $\mathcal{Z}(\mu)$ are given.
\end{abstract}

1. Introduction. Let $H^{\infty}$ be the Banach algebra of bounded analytic functions on the open unit disk $D$. We denote by $\mathcal{M}=M\left(H^{\infty}\right)$ the maximal ideal space of $H^{\infty}$, the space of non-zero multiplicative linear functionals of $H^{\infty}$ with the weak* topology. We think of $D$ as an open subset of $\mathcal{M}$. Identifying a function in $H^{\infty}$ with its Gelfand transform, we regard $H^{\infty}$ as a closed subalgebra of $C(\mathcal{M})$, the space of continuous functions on $\mathcal{M}$. Identifying a function in $H^{\infty}$ with its boundary function, we also view $H^{\infty}$ as an (essential) supremum norm closed subalgebra of $L^{\infty}$, the usual Lebesgue space on the unit circle $\partial D$. We may consider the maximal ideal space $M\left(L^{\infty}\right)$ of $L^{\infty}$ to be a subset of $\mathcal{M}$, and it is known that $M\left(L^{\infty}\right)$ is the Shilov boundary of $H^{\infty}$ (see [10]). For a point $x \in \mathcal{M}$, there exists a probability measure $\mu_{x}$ on $M\left(L^{\infty}\right)$ such that $f(x)=\int_{M\left(L^{\infty}\right)} f d \mu_{x}$ for every $f \in H^{\infty}$. We denote by $\operatorname{supp} \mu_{x}$ the closed support set of $\mu_{x}$. A function $f$ in $H^{\infty}$ is called inner if $|f|=1$ on $M\left(L^{\infty}\right)$. For a function $f$ in $H^{\infty}$, we use the following notations:

$$
\{|f|<1\}=\{x \in \mathcal{M} \backslash D ;|f(x)|<1\}, \quad Z(f)=\{x \in \mathcal{M} \backslash D ; f(x)=0\} .
$$

2000 Mathematics Subject Classification: Primary 46J15.

Key words and phrases: common zero set, singular inner function.

Supported by Grant-in-Aid for Scientific Research (No. 10440039), Ministry of Education, Science and Culture. 
Note that these are subsets of $\mathcal{M} \backslash D$. For $\zeta \in \partial D$, let $\mathcal{M}_{\zeta}=\{x \in \mathcal{M} ; z(x)=\zeta\}$, where $z$ is the identity function on $D$. For a subset $E$ of $\mathcal{M}$, we denote by $\bar{E}$ its weak* closure in $\mathcal{M}$.

For a sequence $\left\{z_{n}\right\}_{n}$ in $D$ with $\sum_{n=1}^{\infty}\left(1-\left|z_{n}\right|\right)<\infty$, there is the associated Blaschke product

$$
b(z)=\prod_{n=1}^{\infty} \frac{-\bar{z}_{n}}{\left|z_{n}\right|} \frac{z-z_{n}}{1-\bar{z}_{n} z}, \quad z \in D .
$$

Blaschke products are typical inner functions. Moreover if for every bounded sequence $\left\{a_{n}\right\}_{n}$ of complex numbers there exists $f \in H^{\infty}$ such that $f\left(z_{n}\right)=$ $a_{n}$ for every $n$, then both $\left\{z_{n}\right\}_{n}$ and the associated Blaschke product $b$ are called interpolating. In this case, we have $Z(b)=\overline{\left\{z_{n}\right\}_{n}} \backslash\left\{z_{n}\right\}_{n}$ (see [10, p. 205]). We denote by $S(b)$ the set of cluster points of $\left\{z_{n}\right\}_{n}$ in the closed unit disk.

For $x, y \in \mathcal{M}$, let

$$
\begin{aligned}
\varrho(x, y) & =\sup \left\{|f(y)| ; f \in H^{\infty}, f(x)=0,\|f\|_{\infty}=1\right\}, \\
P(x) & =\{w \in \mathcal{M} ; \varrho(x, w)<1\} .
\end{aligned}
$$

The set $P(x)$ is called the Gleason part containing $x$. When $P(x)=\{x\}$, both $x$ and $P(x)$ are called trivial. We denote by $G$ the set of non-trivial points in $\mathcal{M}$. In [11], Hoffman proved that $G \backslash D$ is the set of points $x$ in $\mathcal{M} \backslash D$ such that $b(x)=0$ for some interpolating Blaschke product $b$, and $G$ is open in $\mathcal{M}$. See [11] for the study of the structure of $\mathcal{M}$ and $G$.

Let $M_{\mathrm{s}}^{+}$be the set of bounded positive (non-zero) measures on $\partial D$ singular with respect to the Lebesgue measure on $\partial D$. For $\mu \in M_{\mathrm{s}}^{+}$, we denote by $\operatorname{supp} \mu$ the closed support set of $\mu$ and by $\|\mu\|$ the total variation norm of $\mu$. We also denote by $M_{\mathrm{s}, \mathrm{d}}^{+}$and $M_{\mathrm{s}, \mathrm{c}}^{+}$the sets of discrete and continuous measures in $M_{\mathrm{s}}^{+}$, respectively. For $\zeta \in \partial D$, let $\delta_{\zeta}$ be the unit point mass at $\zeta$. For $\mu, \lambda \in M_{\mathrm{s}}^{+}$, we write $\mu \ll \lambda$ if $\mu$ is absolutely continuous with respect to $\lambda$, and $\mu \perp \lambda$ if $\mu$ and $\lambda$ are mutually singular; moreover, $\mu \wedge \lambda$ is the lower bound of $\mu$ and $\lambda$. For $\mu, \nu \in M_{\mathrm{s}}^{+}$, we write $\mu \sim \nu$ if $\mu$ and $\nu$ are equivalent, that is, $\mu \ll \nu$ and $\nu \ll \mu$. For each $\mu \in M_{\mathrm{s}}^{+}$, let

$$
\psi_{\mu}(z)=\exp \left(-\int_{\partial D} \frac{e^{i \theta}+z}{e^{i \theta}-z} d \mu\left(e^{i \theta}\right)\right), \quad z \in D .
$$

Then $\psi_{\mu}$ is called a singular inner function; it may be extended continuously on $\partial D \backslash \operatorname{supp} \mu$ and $\left|\psi_{\mu}\right|=1$ on $\mathcal{M}_{\zeta}$ for $\zeta \notin \operatorname{supp} \mu$ (see $[5,10]$ ). When $\mu \sim \nu$, we say that $\psi_{\mu}$ and $\psi_{\nu}$ are equivalent singular inner functions. We have

$$
\left|\psi_{\mu}(z)\right|=\exp \left(-\int_{\partial D} P_{z}\left(e^{i \theta}\right) d \mu\left(e^{i \theta}\right)\right), \quad z \in D
$$


where $P_{z}$ is the Poisson kernel. We put

$$
\mathcal{Z}(\mu)=\bigcap_{\left\{\nu \in M_{\mathrm{s}}^{+} ; \nu \sim \mu\right\}} Z\left(\psi_{\nu}\right), \quad \mathcal{W}(\mu)=\bigcap_{\left\{\nu \in M_{\mathrm{s}}^{+} ; \nu \sim \mu\right\}}\left\{\left|\psi_{\nu}\right|<1\right\} .
$$

Then $\mathcal{Z}(\mu) \subset \mathcal{W}(\mu)$. In [13], the author proved that if $\mu, \lambda \in M_{\mathrm{s}, \mathrm{d}}^{+}$and $\mu \perp \lambda$, then $\mathcal{W}(\mu) \cap \mathcal{W}(\lambda)=\emptyset$.

The purpose of this paper is to study $\mathcal{Z}(\mu)$ and $\mathcal{W}(\mu)$ for $\mu \in M_{\mathrm{s}}^{+}$. The motivation for this study comes from [12] and [14]. In [12], the author studied certain properties of Blaschke products, and in [14] similar properties for singular inner functions. In Section 2, we prove that if $\mu, \lambda \in M_{\mathrm{s}}^{+}$with $\mu \perp \lambda$, then there are $\mu_{0}, \lambda_{0} \in M_{\mathrm{s}}^{+}$such that $\mu_{0} \sim \mu, \lambda_{0} \sim \lambda$, and $\left\{\left|\psi_{\mu_{0}}\right|<1\right\}$ $\cap\left\{\left|\psi_{\lambda_{0}}\right|<1\right\}=\emptyset$. Then we get $\mathcal{W}(\mu) \cap \mathcal{W}(\lambda)=\emptyset$ and $\mathcal{Z}(\mu) \cap \mathcal{Z}(\lambda)=\emptyset$. Hence $\mathcal{Z}(\mu)$ is the set in $\mathcal{M} \backslash D$ related to the class of measures equivalent to $\mu$. From the point of view of the study of measures on $\partial D$, the set $\mathcal{Z}(\mu)$ is interesting and important. In Section 3, we prove that

$$
\mathcal{W}(\mu)=\mathcal{Z}(\mu) \cup \bigcup_{\{\zeta \in \partial D ; \mu(\{\zeta\}) \neq 0\}}\left\{\left|\psi_{\delta_{\zeta}}\right|<1\right\} .
$$

Hence if $\mu \in M_{\mathrm{s}, \mathrm{c}}^{+}$, then $\mathcal{W}(\mu)=\mathcal{Z}(\mu)$. Moreover, we prove that for $\zeta \in \partial D$, if $\mu(\{\zeta\})=0$ then

$$
\mathcal{Z}(\mu) \subset \overline{\bigcup_{\{\xi \in \partial D ; \xi \neq \zeta\}} \mathcal{M}_{\xi}}
$$

In Section 4 , we prove that for $\zeta \in \partial D$ there exists a Blaschke product $b$ such that $S(b)=\{\zeta\}$ and $\mathcal{Z}(\mu) \cap \overline{\{|b|<1\}}=\emptyset$ for every $\mu \in M_{\mathrm{s}}^{+}$. Also we show that for every Blaschke product $b$ with $S(b)=\partial D$ there exists $\mu \in M_{\mathrm{s}, \mathrm{c}}^{+}$such that $Z(b) \cap \mathcal{Z}(\mu) \neq \emptyset$.

By [4, p. 162], $Z\left(\psi_{\mu}\right)$ contains a trivial point for every $\mu \in M_{\mathrm{s}}^{+}$. Hence $\mathcal{Z}(\mu)$ contains trivial points too. Let int $\mathcal{Z}(\mu)$ denote the interior of $\mathcal{Z}(\mu)$ in $\mathcal{M} \backslash D$. If $\mu \in M_{\mathrm{s}, \mathrm{c}}^{+}$, we have $Z(b) \not \subset \mathcal{Z}(\mu)$ for every interpolating Blaschke product $b$. This implies that int $\mathcal{Z}(\mu)=\emptyset$. Note that int $Z\left(\psi_{\mu}\right) \neq \emptyset$. Since the set $G$ of non-trivial points is open, one can ask whether $\mathcal{Z}(\mu) \cap G=\emptyset$ or not. To answer this, in Section 5 we study interpolating Blaschke products. For a non-empty closed subset $K$ of $\partial D$ which has Lebesgue measure zero, we construct an interpolating Blaschke product $b_{K}$ with certain properties. In Section 6 , we prove that $Z\left(b_{K}\right) \cap \mathcal{Z}(\mu) \neq \emptyset$ for every $\mu \in M_{\mathrm{s}}^{+}$with $\operatorname{supp} \mu \subset K$. Hence $\mathcal{Z}(\mu)$ contains non-trivial points for every $\mu \in M_{\mathrm{s}}^{+}$.

Let $\mu \in M_{\mathrm{s}}^{+}$. We denote by $M\left(L^{\infty}(\mu)\right)$ the maximal ideal space of the Banach algebra $L^{\infty}(\mu)$. In Section 6, we establish the existence of a natural map $\Phi_{\mu}$ from $M\left(L^{\infty}(\mu)\right)$ to the family of closed subsets of $\mathcal{Z}(\mu)$ such that

$$
\mathcal{Z}(\mu)=\bigcup_{x \in M\left(L^{\infty}(\mu)\right)} \Phi_{\mu}(x)
$$


and $\Phi_{\mu}(x) \cap \Phi_{\mu}(y)=\emptyset$ if $x \neq y$. Hence we may think of $\left\{\Phi_{\mu}(x) ; x \in\right.$ $\left.M\left(L^{\infty}(\mu)\right)\right\}$ as an atomic decomposition of the measure $\mu$ in $\mathcal{M} \backslash D$ in some sense. Also we prove that every $\Phi_{\mu}(x)$ contains non-trivial points.

2. Mutually singular measures. For a subset $E$ of $D \cup \partial D$, we denote by $\mathrm{cl} E$ the closure of $E$ in the complex plane. In this section, we prove that $\mathcal{W}(\mu) \cap \mathcal{W}(\lambda)=\emptyset$ if $\mu, \lambda \in M_{\mathrm{s}}^{+}$and $\mu \perp \lambda$. First, we prove the following theorem.

Theorem 2.1. Let $\mu, \lambda \in M_{s}^{+}$and $\mu \perp \lambda$. Then there exist $\mu_{0}, \lambda_{0} \in M_{\mathrm{s}}^{+}$ such that $\mu_{0} \sim \mu, \lambda_{0} \sim \lambda$, and $\left\{\left|\psi_{\mu_{0}}\right|<1\right\} \cap\left\{\left|\psi_{\lambda_{0}}\right|<1\right\}=\emptyset$.

Proof. Since $\mu \perp \lambda$, there exists a measurable subset $A \subset \partial D$ such that $\mu(A)=\|\mu\|$ and $\lambda(\partial D \backslash A)=\|\lambda\|$. By the regularity of the measures, there exist sequences $\left\{\mu_{n}\right\}_{n}$ and $\left\{\lambda_{n}\right\}_{n}$ of measures in $M_{\mathrm{s}}^{+}$such that supp $\mu_{n} \subset A$, $\operatorname{supp} \lambda_{n} \subset \partial D \backslash A$, and

$$
\mu=\sum_{n=1}^{\infty} \mu_{n}, \quad \lambda=\sum_{n=1}^{\infty} \lambda_{n} .
$$

Then

$$
\operatorname{supp} \mu_{n} \cap \operatorname{supp} \lambda_{k}=\emptyset \quad \text { for all } n, k .
$$

Let $\left\{\delta_{n}\right\}_{n}$ be a sequence of numbers such that

$$
0<\delta_{n}<1, \quad \prod_{n=1}^{\infty} \delta_{n}>0 .
$$

For each $0<s<1$, let

$$
U_{\mu_{n}}(s)=\left\{z \in D ;\left|\psi_{\mu_{n}}(z)\right|<s\right\}, \quad U_{\lambda_{n}}(s)=\left\{z \in D ;\left|\psi_{\lambda_{n}}(z)\right|<s\right\} .
$$

Then $U_{\mu_{n}}\left(s_{1}\right) \subset U_{\mu_{n}}\left(s_{2}\right)$ if $s_{1}<s_{2}$, and

$$
\bigcap_{0<s<1} \operatorname{cl} U_{\mu_{n}}(s)=\operatorname{supp} \mu_{n}, \quad \bigcap_{0<s<1} \operatorname{cl} U_{\lambda_{n}}(s)=\operatorname{supp} \lambda_{n} .
$$

Hence by (2.2), we have

$$
\sup _{z \in U_{\mu_{k}}(s)}\left|\psi_{\lambda_{n}}(z)\right| \rightarrow 1, \quad \sup _{z \in U_{\lambda_{k}}(s)}\left|\psi_{\mu_{n}}(z)\right| \rightarrow 1 \quad \text { as } s \rightarrow 0 \text { for all } n, k .
$$

Then by induction, we may take $\left\{s_{n}\right\}_{n}$ and $\left\{t_{n}\right\}_{n}$ such that

$$
U_{\mu_{n}}\left(s_{n}\right) \cap U_{\lambda_{k}}\left(t_{k}\right)=\emptyset \text { for all } n, k,
$$




$$
\begin{aligned}
& \left|\prod_{j=1}^{n} \psi_{\lambda_{j}}\right| \geq \delta_{n} \quad \text { on } \bigcup_{k=n}^{\infty} U_{\mu_{k}}\left(s_{k}\right), \\
& \left|\prod_{j=1}^{n} \psi_{\mu_{j}}\right| \geq \delta_{n} \quad \text { on } \bigcup_{k=n}^{\infty} U_{\lambda_{k}}\left(t_{k}\right) .
\end{aligned}
$$

Next, let $\left\{a_{n}\right\}_{n}$ and $\left\{b_{n}\right\}_{n}$ be sequences of numbers satisfying

$$
\begin{gathered}
0<a_{n}<1, \quad 0<b_{n}<1, \\
s_{n}^{a_{n}} \geq \delta_{n}, \quad t_{n}^{b_{n}} \geq \delta_{n} \quad \text { for every } n .
\end{gathered}
$$

Let

$$
\mu_{0}=\sum_{n=1}^{\infty} a_{n} \mu_{n}, \quad \lambda_{0}=\sum_{n=1}^{\infty} b_{n} \lambda_{n} .
$$

Then by $(2.1)$ and $(2,7), \mu_{0}, \lambda_{0} \in M_{\mathrm{s}}^{+}, \mu_{0} \sim \mu$, and $\lambda_{0} \sim \lambda$.

For $z \in D \backslash \bigcup_{j=1}^{\infty} U_{\mu_{j}}\left(s_{j}\right)$, we have

$$
\begin{aligned}
\left|\psi_{\mu_{0}}(z)\right| & =\prod_{j=1}^{k}\left|\psi_{\mu_{j}}(z)\right|^{a_{j}} \prod_{j=k+1}^{\infty}\left|\psi_{\mu_{j}}(z)\right|^{a_{j}} & & \text { by }(2.9) \\
& \geq \prod_{j=1}^{k}\left|\psi_{\mu_{j}}(z)\right| \prod_{j=k+1}^{\infty} s_{j}^{a_{j}} & & \text { by }(2.4) \\
& \geq \prod_{j=1}^{k}\left|\psi_{\mu_{j}}(z)\right| \prod_{j=k+1}^{\infty} \delta_{j} & & \text { by }(2.8) .
\end{aligned}
$$

Hence

$$
\left|\psi_{\mu_{0}}\right| \geq \prod_{j=1}^{k}\left|\psi_{\mu_{j}}\right| \prod_{j=k+1}^{\infty} \delta_{j} \quad \text { on } D \backslash \bigcup_{j=1}^{\infty} U_{\mu_{j}}\left(s_{j}\right) \text { for every } k .
$$

Similarly,

$$
\left|\psi_{\lambda_{0}}\right| \geq \prod_{j=1}^{k}\left|\psi_{\lambda_{j}}\right| \prod_{j=k+1}^{\infty} \delta_{j} \quad \text { on } D \backslash \bigcup_{j=1}^{\infty} U_{\lambda_{j}}\left(t_{j}\right) \text { for every } k .
$$

Now suppose that $\left\{\left|\psi_{\mu_{0}}\right|<1\right\} \cap\left\{\left|\psi_{\lambda_{0}}\right|<1\right\} \neq \emptyset$. Then by the corona theorem [3], there exist $0<\delta<1$ and a sequence $\left\{z_{n}\right\}_{n}$ in $D$ such that $\left|z_{n}\right| \rightarrow 1$ and

$$
\left|\psi_{\mu_{0}}\left(z_{n}\right)\right|<\delta, \quad\left|\psi_{\lambda_{0}}\left(z_{n}\right)\right|<\delta \quad \text { for every } n .
$$


By (2.3), there exists a positive integer $k_{0}$ such that

$$
\prod_{j=k_{0}+1}^{\infty} \delta_{j}>\delta^{1 / 2}
$$

Considering a subsequence of $\left\{z_{n}\right\}_{n}$, we may further assume that either

$$
\begin{gathered}
z_{n} \in\left(D \backslash \bigcup_{j=1}^{\infty} U_{\mu_{j}}\left(s_{j}\right)\right) \cap\left(D \backslash \bigcup_{j=1}^{\infty} U_{\lambda_{j}}\left(t_{j}\right)\right) \quad \text { for every } n \\
z_{n} \in \bigcup_{j=1}^{\infty} U_{\mu_{j}}\left(s_{j}\right) \quad \text { for every } n
\end{gathered}
$$

or

$$
z_{n} \in \bigcup_{j=1}^{\infty} U_{\lambda_{j}}\left(t_{j}\right) \quad \text { for every } n .
$$

For each case we shall obtain a contradiction.

First, suppose that (2.14) holds. By (2.10), (2.12), and (2.13),

$$
\delta>\prod_{j=1}^{k_{0}}\left|\psi_{\mu_{j}}\left(z_{n}\right)\right| \prod_{j=k_{0}+1}^{\infty} \delta_{j}>\delta^{1 / 2} \prod_{j=1}^{k_{0}}\left|\psi_{\mu_{j}}\left(z_{n}\right)\right| \quad \text { for every } n .
$$

Then

$$
\prod_{j=1}^{k_{0}}\left|\psi_{\mu_{j}}\left(z_{n}\right)\right| \leq \delta^{1 / 2}<1 \quad \text { for every } n
$$

Similarly,

$$
\prod_{j=1}^{k_{0}}\left|\psi_{\lambda_{j}}\left(z_{n}\right)\right| \leq \delta^{1 / 2}<1 \quad \text { for every } n
$$

Hence

$$
\operatorname{cl}\left\{z_{n}\right\}_{n} \backslash\left\{z_{n}\right\}_{n} \subset\left(\bigcup_{j=1}^{k_{0}} \operatorname{supp} \mu_{j}\right) \cap\left(\bigcup_{j=1}^{k_{0}} \operatorname{supp} \lambda_{j}\right) .
$$

But this contradicts (2.2). Therefore (2.14) does not occur.

Next, suppose that (2.15) holds. Then by (2.5),

$$
\left\{z_{n}\right\}_{n} \subset D \backslash \bigcup_{j=1}^{\infty} U_{\lambda_{j}}\left(t_{j}\right)
$$

Taking a subsequence of $\left\{z_{n}\right\}_{n}$, we may further assume that either

$$
\left\{z_{n}\right\}_{n} \subset \bigcup_{j=1}^{m} U_{\mu_{j}}\left(s_{j}\right) \quad \text { for some } m \geq k_{0},
$$


or

$$
\left\{z_{n}\right\}_{n} \cap \bigcup_{j=1}^{m} U_{\mu_{j}}\left(s_{j}\right) \quad \text { is a finite set for every } m .
$$

Suppose that (2.18) holds. Then by (2.4), we have

$$
\prod_{j=1}^{m}\left|\psi_{\mu_{j}}\left(z_{n}\right)\right|<\max _{1 \leq j \leq m} s_{j}<1 \quad \text { for every } n \text {. }
$$

Hence

$$
\operatorname{cl}\left\{z_{n}\right\}_{n} \backslash\left\{z_{n}\right\}_{n} \subset \bigcup_{j=1}^{m} \operatorname{supp} \mu_{j}
$$

By (2.11), (2.12), (2.13), and (2.17),

$$
\delta>\left|\psi_{\lambda_{0}}\left(z_{n}\right)\right| \geq \prod_{j=1}^{k_{0}}\left|\psi_{\lambda_{j}}\left(z_{n}\right)\right| \prod_{j=k_{0}+1}^{\infty} \delta_{j}>\delta^{1 / 2} \prod_{j=1}^{k_{0}}\left|\psi_{\lambda_{j}}\left(z_{n}\right)\right| .
$$

Thus we have

$$
\prod_{j=1}^{k_{0}}\left|\psi_{\lambda_{j}}\left(z_{n}\right)\right|<\delta^{1 / 2}<1 \quad \text { for every } n
$$

Therefore

$$
\operatorname{cl}\left\{z_{n}\right\}_{n} \backslash\left\{z_{n}\right\}_{n} \subset \bigcup_{j=1}^{k_{0}} \operatorname{supp} \lambda_{j}
$$

Hence (2.20) and (2.21) contradict (2.2).

Next, suppose that (2.19) holds. Then for each $k$, we have

$$
\begin{aligned}
\liminf _{n \rightarrow \infty}\left|\psi_{\lambda_{0}}\left(z_{n}\right)\right| & \geq \liminf _{n \rightarrow \infty} \prod_{j=1}^{k}\left|\psi_{\lambda_{j}}\left(z_{n}\right)\right| \prod_{j=k+1}^{\infty} \delta_{j} \quad \text { by }(2.11) \text { and }(2.17) \\
& \geq \prod_{j=k}^{\infty} \delta_{j} \text { by }(2.6),(2.15), \text { and }(2.19) .
\end{aligned}
$$

Thus by (2.3), we have $\left|\psi_{\lambda_{0}}\left(z_{n}\right)\right| \rightarrow 1$ as $n \rightarrow \infty$. This contradicts (2.12). Therefore (2.15) does not occur.

Similarly, we may prove that (2.16) does not occur. Thus we get our assertion.

As an application of Theorem 2.1, we have the following.

TheOREM 2.2. Let $\mu, \lambda \in M_{\mathrm{s}}^{+}$be such that $\mu \perp \lambda$. Then $\mathcal{W}(\mu) \cap \mathcal{W}(\lambda)$ $=\emptyset$, and consequently, $\mathcal{Z}(\mu) \cap \mathcal{Z}(\lambda)=\emptyset$. 
This theorem says that the singularity of measures on $\partial D$ may be represented in the maximal ideal space $\mathcal{M}$ of $H^{\infty}$ as disjoint closed subsets. So to study the behavior of singular inner functions, it is important to study the sets $\mathcal{Z}(\mu)$.

3. $\mathcal{Z}(\mu)$ and $\mathcal{W}(\mu)$. Recall that for $\mu \in M_{\mathrm{s}}^{+}$,

$$
\mathcal{Z}(\mu)=\bigcap_{\left\{\nu \in M_{\mathrm{s}}^{+} ; \nu \sim \mu\right\}} Z\left(\psi_{\nu}\right), \quad \mathcal{W}(\mu)=\bigcap_{\left\{\nu \in M_{\mathrm{s}}^{+} ; \nu \sim \mu\right\}}\left\{\left|\psi_{\nu}\right|<1\right\} .
$$

Thus $\mathcal{Z}(\mu) \subset \mathcal{W}(\mu)$ and $\mathcal{W}(\mu)$ is a subset of $\mathcal{M} \backslash\left(D \cup M\left(L^{\infty}\right)\right)$. In this section, we study the properties of $\mathcal{Z}(\mu)$ and $\mathcal{W}(\mu)$. We note that if $\mu, \lambda \in$ $M_{\mathrm{s}}^{+}$and $\mu \sim \lambda$, then $\mathcal{Z}(\mu)=\mathcal{Z}(\lambda)$ and $\mathcal{W}(\mu)=\mathcal{W}(\lambda)$.

First, we prove the following.

Theorem 3.1. Let $\mu \in M_{\mathrm{s}}^{+}$and $\zeta \in \operatorname{supp} \mu$. Then $\mathcal{Z}(\mu) \cap \mathcal{M}_{\zeta} \neq \emptyset$, and consequently, $\mathcal{Z}(\mu) \neq \emptyset$.

To prove this, we use the following lemma.

Lemma 3.2. Let $\mu \in M_{\mathrm{s}}^{+}$and $E$ be a closed subset of $\mathcal{M}$ such that $\mathcal{Z}(\mu) \cap E=\emptyset$. Then there exists $\nu \in M_{\mathrm{s}}^{+}$such that $\nu \sim \mu$ and $Z\left(\psi_{\nu}\right) \cap E=\emptyset$.

Proof. By our assumption, there exist $\nu_{1}, \ldots, \nu_{n} \in M_{\mathrm{s}}^{+}$such that $\nu_{j} \sim \mu$ and

$$
\sum_{j=1}^{n}\left|\psi_{\nu_{j}}\right|>0 \quad \text { on } E \text {. }
$$

Let $\nu$ be the lower bound of $\left\{\nu_{j}\right\}_{j=1}^{n}$, that is, $\nu=\bigwedge_{j=1}^{n} \nu_{j}$. Then $\nu \neq 0$ and $\nu \sim \mu$. Since $\nu \leq \nu_{j}$, we have $\left|\psi_{\nu_{j}}\right| \leq\left|\psi_{\nu}\right|$ on $\mathcal{M}$. Hence by (3.1), $0<\left|\psi_{\nu}\right|$ on $E$.

Proof of Proposition 3.1. Let $\nu \in M_{\mathrm{s}}^{+}$and $\nu \sim \mu$. Since $\zeta \in \operatorname{supp} \nu$, it follows that $Z\left(\psi_{\nu}\right) \cap \mathcal{M}_{\zeta} \neq \emptyset$ (see [5, p. 76]). By Lemma 3.2, we have $\mathcal{Z}(\mu) \cap \mathcal{M}_{\zeta} \neq \emptyset$.

The following lemma lists elementary properties of $\mathcal{Z}(\mu)$ and $\mathcal{W}(\mu)$.

Lemma 3.3. Let $\mu_{1}, \mu_{2} \in M_{\mathrm{s}}^{+}$.

(i) If $\mu_{1} \perp \mu_{2}$, then $\mathcal{Z}\left(\mu_{1}+\mu_{2}\right)=\mathcal{Z}\left(\mu_{1}\right) \cup \mathcal{Z}\left(\mu_{1}\right)$ and $\mathcal{W}\left(\mu_{1}+\mu_{2}\right)=$ $\mathcal{W}\left(\mu_{1}\right) \cup \mathcal{W}\left(\mu_{1}\right)$.

(ii) If $\mu_{1} \ll \mu_{2}$, then $\mathcal{Z}\left(\mu_{1}\right) \subset \mathcal{Z}\left(\mu_{2}\right)$ and $\mathcal{W}\left(\mu_{1}\right) \subset \mathcal{W}\left(\mu_{2}\right)$.

(iii) $\mathcal{Z}\left(\mu_{1}+\mu_{2}\right)=\mathcal{Z}\left(\mu_{1}\right) \cup \mathcal{Z}\left(\mu_{2}\right)$ and $\mathcal{W}\left(\mu_{1}+\mu_{2}\right)=\mathcal{W}\left(\mu_{1}\right) \cup \mathcal{W}\left(\mu_{2}\right)$.

(iv) If $\mu_{1} \wedge \mu_{2} \neq 0$, then $\mathcal{Z}\left(\mu_{1} \wedge \mu_{2}\right)=\mathcal{Z}\left(\mu_{1}\right) \cap \mathcal{Z}\left(\mu_{2}\right)$ and $\mathcal{W}\left(\mu_{1} \wedge \mu_{2}\right)=$ $\mathcal{W}\left(\mu_{1}\right) \cap \mathcal{W}\left(\mu_{2}\right)$. 
Proof. We only prove the properties of $\mathcal{Z}(\mu)$; those of $\mathcal{W}(\mu)$ are established similarly.

(i) Suppose that $\mu_{1} \perp \mu_{2}$. Let $\nu \in M_{\mathrm{s}}^{+}$. Then $\nu \sim \mu_{1}+\mu_{2}$ if and only if $\nu=\nu_{1}+\nu_{2}$ for some $\nu_{1}, \nu_{2} \in M_{\mathrm{s}}^{+}$with $\nu_{1} \sim \mu_{1}$ and $\nu_{2} \sim \mu_{2}$. Since $\psi_{\nu_{1}+\nu_{2}}=\psi_{\nu_{1}} \psi_{\nu_{2}}$, we have $Z\left(\psi_{\nu_{1}+\nu_{2}}\right)=Z\left(\psi_{\nu_{1}}\right) \cup Z\left(\psi_{\nu_{2}}\right)$. Then by Theorem $2.2, \mathcal{Z}\left(\mu_{1}+\mu_{2}\right)=\mathcal{Z}\left(\mu_{1}\right) \cup \mathcal{Z}\left(\mu_{2}\right)$.

(ii) Suppose that $\mu_{1} \ll \mu_{2}$. Then $\mu_{2}=\nu_{1}+\nu_{2}$, where $\nu_{1} \sim \mu_{1}$ and $\nu_{1} \perp \nu_{2}$. Hence by (i), $\mathcal{Z}\left(\mu_{1}\right)=\mathcal{Z}\left(\nu_{1}\right) \subset \mathcal{Z}\left(\nu_{1}\right) \cup \mathcal{Z}\left(\nu_{2}\right)=\mathcal{Z}\left(\mu_{2}\right)$.

(iii) By (ii), we have $\mathcal{Z}\left(\mu_{1}\right) \cup \mathcal{Z}\left(\mu_{2}\right) \subset \mathcal{Z}\left(\mu_{1}+\mu_{2}\right)$. To prove the reverse inclusion, write $\mu_{1}+\mu_{2}=\nu_{1}+\nu_{2}$, where $\nu_{1}, \nu_{2} \in M_{\mathrm{s}}^{+}$are such that $\nu_{1} \sim \mu_{1}$, $\nu_{1} \perp \nu_{2}$, and $\nu_{2} \ll \mu_{2}$. Then by (i) and (ii),

$$
\mathcal{Z}\left(\mu_{1}+\mu_{2}\right)=\mathcal{Z}\left(\nu_{1}\right) \cup \mathcal{Z}\left(\nu_{2}\right)=\mathcal{Z}\left(\mu_{1}\right) \cup \mathcal{Z}\left(\nu_{2}\right) \subset \mathcal{Z}\left(\mu_{1}\right) \cup \mathcal{Z}\left(\mu_{2}\right) \text {. }
$$

(iv) By (ii), $\mathcal{Z}\left(\mu_{1} \wedge \mu_{2}\right) \subset \mathcal{Z}\left(\mu_{1}\right) \cap \mathcal{Z}\left(\mu_{2}\right)$. Write $\mu_{1}=\nu_{1}+\nu_{2}$, where $\nu_{1} \sim \mu_{1} \wedge \mu_{2}$ and $\nu_{2} \perp \mu_{2}$. Then by (i),

$$
\mathcal{Z}\left(\mu_{1}\right)=\mathcal{Z}\left(\nu_{1}\right) \cup \mathcal{Z}\left(\nu_{2}\right)=\mathcal{Z}\left(\mu_{1} \wedge \mu_{2}\right) \cup \mathcal{Z}\left(\nu_{2}\right) .
$$

By Theorem 2.2, $\mathcal{Z}\left(\nu_{2}\right) \cap \mathcal{Z}\left(\mu_{2}\right)=\emptyset$. By (ii), $\mathcal{Z}\left(\mu_{1} \wedge \mu_{2}\right) \subset \mathcal{Z}\left(\mu_{2}\right)$. Hence

$$
\mathcal{Z}\left(\mu_{1}\right) \cap \mathcal{Z}\left(\mu_{2}\right)=\mathcal{Z}\left(\mu_{1} \wedge \mu_{2}\right) \cap \mathcal{Z}\left(\mu_{2}\right)=\mathcal{Z}\left(\mu_{1} \wedge \mu_{2}\right) .
$$

Proposition 3.4. Let $\mu_{1}, \mu_{2} \in M_{\mathrm{S}}^{+}$. Then $\mu_{1} \ll \mu_{2}$ if and only if $\mathcal{Z}\left(\mu_{1}\right) \subset \mathcal{Z}\left(\mu_{2}\right)$.

Proof. The "only if" part follows from Lemma 3.3(ii). Suppose that $\mu_{1} \nless \mu_{2}$. Write $\mu_{1}=\nu_{1}+\nu_{2}$, where $\nu_{1} \perp \mu_{2}$ and $\nu_{2} \ll \mu_{2}$. Then $\nu_{1} \neq 0$. By Proposition 3.1, we have $\mathcal{Z}\left(\nu_{1}\right) \neq \emptyset$. Since $\nu_{1} \ll \mu_{1}$, Lemma 3.3(ii) yields $\mathcal{Z}\left(\nu_{1}\right) \subset \mathcal{Z}\left(\mu_{1}\right)$. Since $\nu_{1} \perp \mu_{2}$, by Theorem 2.2 we have $\mathcal{Z}\left(\nu_{1}\right) \cap \mathcal{Z}\left(\mu_{2}\right)=\emptyset$. Thus we get $\mathcal{Z}\left(\mu_{1}\right) \not \subset \mathcal{Z}\left(\mu_{2}\right)$.

The following shows a relation between $\mathcal{W}(\mu)$ and $\mathcal{Z}(\mu)$.

Theorem 3.5. Let $\mu \in M_{\mathrm{s}}^{+}$. Then

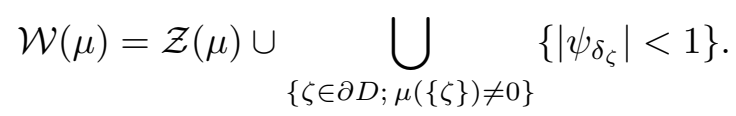

Proof. The $\supset$ inclusion follows from the definition of $\mathcal{W}(\mu)$. To prove the reverse inclusion, let

$$
x \in \mathcal{W}(\mu) \backslash \bigcup_{\{\zeta \in \partial D ; \mu(\{\zeta\}) \neq 0\}}\left\{\left|\psi_{\delta_{\zeta}}\right|<1\right\} .
$$

It is sufficient to prove that $x \in \mathcal{Z}(\mu)$. Suppose not. Then there exists $\nu \in M_{\mathrm{s}}^{+}$such that $\nu \sim \mu$ and $\psi_{\nu}(x) \neq 0$. We may assume that $x \in \mathcal{M}_{1}$.

First, suppose that $\mu(\{1\})=0$. Let $I_{0}=\partial D$ and $I_{n}=\left\{e^{i \theta} ;-1 / n \leq \theta \leq\right.$ $1 / n\}$ for every positive integer $n$. Set $\nu_{n}=\nu_{\mid\left(I_{n-1} \backslash I_{n}\right)}$. Then $\nu=\sum_{n=1}^{\infty} \nu_{n}$. 
Let

$$
\nu_{0}=\sum_{n=1}^{\infty} \nu_{n} / n
$$

Then $\nu_{0} \sim \nu \sim \mu$ and

$$
k \nu_{0} \leq \nu+\sum_{n=1}^{k} k \nu_{n} \quad \text { for all } k .
$$

Since $\operatorname{supp} \nu_{n} \subset \operatorname{cl}\left(I_{n-1} \backslash I_{n}\right)$, it follows that $1 \notin \operatorname{supp} \nu_{n}$. Hence $\left|\psi_{\nu_{n}}\right|=1$ on $\mathcal{M}_{1}$ for every $n$. Since $x \in \mathcal{M}_{1}$, by (3.3),

$$
\left|\psi_{\nu}(x)\right|=\left|\psi_{\nu}(x)\right| \prod_{n=1}^{k}\left|\psi_{\nu_{n}}(x)\right|^{k} \leq\left|\psi_{\nu_{0}}(x)\right|^{k} \quad \text { for all } k .
$$

Since $\psi_{\nu}(x) \neq 0$, we have $\left|\psi_{\nu_{0}}(x)\right|=1$, so that $x \notin \mathcal{W}(\mu)$. This contradicts (3.2). Thus if $\mu(\{1\})=0$, then $x \in \mathcal{Z}(\mu)$.

Next, suppose that $\mu(\{1\})=c>0$. Write $\mu=c \delta_{1}+\mu_{1}$, where $\mu_{1} \perp \delta_{1}$. Then by Lemma 3.3(i), $\mathcal{W}(\mu)=\left\{\left|\psi_{\delta_{1}}\right|<1\right\} \cup \mathcal{W}\left(\mu_{1}\right)$, so that we may rewrite condition (3.2) as

$$
x \in \mathcal{W}\left(\mu_{1}\right) \backslash \bigcup_{\left\{\zeta \in \partial D ; \mu_{1}(\{\zeta\}) \neq 0\right\}}\left\{\left|\psi_{\delta_{\zeta}}\right|<1\right\} .
$$

By the previous paragraph, $x \in \mathcal{Z}\left(\mu_{1}\right)$. By Lemma 3.3(ii), $\mathcal{Z}\left(\mu_{1}\right) \subset \mathcal{Z}(\mu)$. Hence $x \in \mathcal{Z}(\mu)$.

Corollary 3.6. Let $\mu \in M_{\mathrm{S}}^{+}$and $\zeta \in \partial D$. If $\mu(\{\zeta\})=0$, then $\mathcal{Z}(\mu) \cap$ $\mathcal{M}_{\zeta}=\mathcal{W}(\mu) \cap \mathcal{M}_{\zeta}$.

Proposition 3.7. Let $\mu \in M_{\mathrm{S}}^{+}$and $E$ be a closed subset of $\partial D$. Let $A$ be an $F_{\sigma}$-subset of $\mathcal{M}$ such that $A \cap \overline{\bigcup_{\xi \in \partial D \backslash E} \mathcal{M}_{\xi}}=\emptyset$. If $\mu(E)=0$, then there exists $\nu \in M_{\mathrm{S}}^{+}$such that $\nu \sim \mu$ and $\left|\psi_{\nu}\right|=1$ on $A$.

Proof. By our assumption, $A=\bigcup_{j=1}^{\infty} A_{j}$, where $A_{j}$ is a closed set. Then there is a sequence $\left\{U_{j}\right\}_{j}$ of open subsets of $\mathcal{M}$ such that

$$
A_{j} \subset U_{j}, \quad \bar{U}_{j} \cap \overline{\bigcup_{\xi \in \partial D \backslash E} \mathcal{M}_{\xi}}=\emptyset \quad \text { for every } j .
$$

Let $I_{0}=\partial D$ and $\left\{I_{n}\right\}_{n}$ be a sequence of open subsets of $\partial D$ such that $I_{n} \subset I_{n-1}$ and $\bigcap_{n=1}^{\infty} I_{n}=E$. Set $\mu_{n}=\mu_{\mid\left(I_{n-1} \backslash I_{n}\right)}$. Since $\mu(E)=0$, we have $\mu=\sum_{n=1}^{\infty} \mu_{n}$. Since $E \cap \operatorname{supp} \mu_{n}=\emptyset$, it follows that $\left|\psi_{\mu_{n}}\right|=1$ on $\bigcup_{\zeta \in E} \mathcal{M}_{\zeta}$. Then by (3.4), $\bar{U}_{j} \backslash D \subset \bigcup_{\zeta \in E} \mathcal{M}_{\zeta}$. Hence for every $n$ and $j$,

$$
\left|\psi_{\mu_{n}}(z)\right| \rightarrow 1 \quad \text { as }|z| \rightarrow 1 \text { and } z \in U_{j} \cap D .
$$


Let $\left\{\varepsilon_{n}\right\}_{n}$ be a sequence of positive numbers such that

$$
\prod_{n=1}^{\infty} \varepsilon_{n}>0, \quad 0<\varepsilon_{n}<1 \quad \text { for every } n .
$$

Then by (3.5), there exists a sequence $\left\{a_{n}\right\}_{n}$ of positive numbers such that $0<a_{n}<1$ and

$$
\left|\psi_{\mu_{n}}(z)\right|^{a_{n}} \geq \varepsilon_{n} \quad \text { on } U_{j} \cap D \text { for } 1 \leq j \leq n .
$$

Let

$$
\nu=\sum_{n=1}^{\infty} a_{n} \mu_{n} .
$$

Then $\nu \in M_{\mathrm{s}}^{+}, \nu \sim \mu$, and for any positive integers $j$ and $m$, we have

$$
\begin{aligned}
\liminf _{|z| \rightarrow 1, z \in U_{j} \cap D}\left|\psi_{\nu}(z)\right| & =\liminf _{|z| \rightarrow 1, z \in U_{j} \cap D} \prod_{n=1}^{\infty}\left|\psi_{\mu_{n}}(z)\right|^{a_{n}} \\
& =\liminf _{|z| \rightarrow 1, z \in U_{j} \cap D} \prod_{n=m}^{\infty}\left|\psi_{\mu_{n}}(z)\right|^{a_{n}} \\
& \geq \prod_{n=m}^{\infty} \varepsilon_{n} \text { by }(3.7) .
\end{aligned}
$$

Hence by (3.6),

$$
\liminf _{|z| \rightarrow 1, z \in U_{j} \cap D}\left|\psi_{\nu}(z)\right|=1 \quad \text { for every } j \text {. }
$$

By the corona theorem and (3.4), $A_{j} \subset \overline{U_{j} \cap D}$. Therefore $\left|\psi_{\nu}\right|=1$ on $A_{j}$ for every $j$. Thus $\left|\psi_{\nu}\right|=1$ on $A$.

Corollary 3.8. Let $\mu \in M_{\mathrm{s}}^{+}$and $E$ be a closed subset of $\partial D$. If $\mu(E)$ $=0$, then

$$
\mathcal{Z}(\mu) \subset \mathcal{W}(\mu) \subset \overline{\bigcup_{\xi \in \partial D \backslash E} \mathcal{M}_{\xi}}
$$

This follows from Proposition 3.7.

Corollary 3.9. Let $\mu \in M_{\mathrm{s}}^{+}$. Then $\mathcal{W}(\mu)=\mathcal{Z}(\mu)$ if and only if $\mu \in M_{\mathrm{s}, \mathrm{c}}^{+}$.

Proof. Suppose that $\mu(\{\zeta\})>0$ for some $\zeta \in \partial D$. Write $\mu=a \delta_{\zeta}+\mu_{1}$, where $\mu_{1}(\{\zeta\})=0$. Then by Lemma 3.3 ,

$$
\mathcal{W}(\mu)=\left\{\left|\psi_{\delta_{\zeta}}\right|<1\right\} \cup \mathcal{W}\left(\mu_{1}\right), \quad \mathcal{Z}(\mu)=Z\left(\psi_{\delta_{\zeta}}\right) \cup \mathcal{Z}\left(\mu_{1}\right) .
$$

Since $\left\{\left|\psi_{\delta_{\zeta}}\right|<1\right\} \cap \overline{\bigcup_{\{\xi \in \partial D ; \xi \neq \zeta\}} \mathcal{M}_{\xi}}=\emptyset$, by Corollary 3.8 we have

$$
\mathcal{W}(\mu) \cap\left\{\left|\psi_{\delta_{\zeta}}\right|<1\right\}=\left\{\left|\psi_{\delta_{\zeta}}\right|<1\right\}, \quad \mathcal{Z}(\mu) \cap\left\{\left|\psi_{\delta_{\zeta}}\right|<1\right\}=Z\left(\psi_{\delta_{\zeta}}\right) .
$$

Therefore $\mathcal{W}(\mu) \neq \mathcal{Z}(\mu)$

The converse follows from Theorem 3.5. 
Corollary 3.10. Let $\mu \in M_{\mathrm{s}, \mathrm{c}}^{+}$be such that $x \in \mathcal{Z}(\mu)$. Let $y \in \mathcal{M} \backslash D$ and $\operatorname{supp} \mu_{x} \subset \operatorname{supp} \mu_{y}$. Then $y \in \mathcal{Z}(\mu)$.

Proof. Let $\nu \in M_{\mathrm{s}}^{+}$and $\nu \sim \mu$. Since $\psi_{\nu}(x)=0$, we have $\left|\psi_{\nu}(y)\right|<1$. Hence $y \in \mathcal{W}(\mu)$. By Corollary 3.9, $y \in \mathcal{Z}(\mu)$.

4. Blaschke products and singular inner functions. Let $b$ be a Blaschke product with zeros $\left\{z_{n}\right\}_{n}$. Recall that $S(b)$ is the set of cluster points of $\left\{z_{n}\right\}_{n}$ in $\partial D$. Then $S(b)$ is the set of points in $\partial D$ to which $b$ may not be extended continuously. Moreover, we have

$$
\{|b|<1\} \cap \overline{\bigcup_{\xi \in \partial D \backslash S(b)} \mathcal{M}_{\xi}}=\emptyset .
$$

There exists a sequence $\left\{p_{n}\right\}_{n}$ of positive integers such that $p_{n} \rightarrow \infty$ as $n \rightarrow \infty$ and

$$
b_{1}(z)=\prod_{n=1}^{\infty}\left(\frac{-\bar{z}_{n}}{\left|z_{n}\right|} \frac{z-z_{n}}{1-\bar{z}_{n} z}\right)^{p_{n}}, \quad z \in D,
$$

is a Blaschke product. Then $S\left(b_{1}\right)=S(b)$ and

$$
\overline{\{|b|<1\}} \subset Z\left(b_{1}\right) \subset\left\{\left|b_{1}\right|<1\right\} .
$$

Hence by (4.1),

$$
\overline{\{|b|<1\}} \cap \overline{\bigcup_{\xi \in \partial D \backslash S(b)} \mathcal{M}_{\xi}}=\emptyset .
$$

Moreover, if

$$
\lim _{k \rightarrow \infty} \prod_{n: n \neq k}\left|\frac{z_{n}-z_{k}}{1-\bar{z}_{k} z_{n}}\right|=1,
$$

then both $b$ and $\left\{z_{n}\right\}_{n}$ are called sparse (or thin).

Suppose that $b$ is sparse. Then

$$
\{|b|<1\}=\bigcup_{x \in Z(b)} P(x)
$$

(see $[7,9])$. For every sequence $\left\{z_{n}\right\}_{n}$ in $D$ with $\left|z_{n}\right| \rightarrow 1$ as $n \rightarrow \infty$, there exists a sparse subsequence of $\left\{z_{n}\right\}_{n}$ (see $[6]$ ).

Lemma 4.1. Let $b$ be a sparse Blaschke product. Let $\varphi$ be an inner function such that $|\varphi|=1$ on $Z(b)$. Then $|\varphi|=1$ on $\overline{\{|b|<1\}}$.

Proof. Let $y \in\{|b|<1\}$. Then by (4.3), $y \in P(x)$ for some $x \in Z(b)$. By $\left[4\right.$, p. 143], $\operatorname{supp} \mu_{y}=\operatorname{supp} \mu_{x}$. Since $|\varphi(x)|=1$, we have $\varphi=\varphi(x)$ on $\operatorname{supp} \mu_{y}$. Hence $\varphi(y)=\int_{M\left(L^{\infty}\right)} \varphi d \mu_{y}=\varphi(x)$. Thus $|\varphi(y)|=1$.

First, we prove the following. 
Proposition 4.2. Let $\mu \in M_{\mathrm{s}}^{+}$. Then there is a sparse Blaschke product $b$ such that $S(b)=\operatorname{supp} \mu$ and $\mathcal{Z}(\mu) \cap \overline{\{|b|<1\}}=\emptyset$.

Proof. Since $\left|\psi_{\mu}\right|=1$ on $M\left(L^{\infty}\right)$, by the corona theorem there exists a sequence $\left\{z_{n}\right\}_{n}$ in $D$ such that $\left|\psi_{\mu}\left(z_{n}\right)\right| \rightarrow 1$ as $n \rightarrow \infty$ and $\operatorname{cl}\left\{z_{n}\right\}_{n} \backslash\left\{z_{n}\right\}_{n}=$ supp $\mu$. Considering a subsequence, we may assume that $\left\{z_{n}\right\}_{n}$ is sparse. Let $b$ be the associated Blaschke product. Then $S(b)=\operatorname{supp} \mu$ and $\left|\psi_{\mu}\right|=1$ on $Z(b)$. By Lemma 4.1, $Z\left(\psi_{\mu}\right) \cap \overline{\{|b|<1\}}=\emptyset$. Thus $\mathcal{Z}(\mu) \cap \overline{\{|b|<1\}}=\emptyset$.

Corollary 4.3. Let $b$ be a Blaschke product. If $\mu \in M_{\mathrm{s}}^{+}$and $\mu(S(b))$ $=0$, then $\mathcal{Z}(\mu) \cap \overline{\{|b|<1\}}=\emptyset$. 3.8 .

Proof. By (4.2), $\overline{\{|b|<1\}} \cap \overline{\bigcup_{\xi \in \partial D \backslash S(b)} \mathcal{M}_{\xi}}=\emptyset$; now apply Corollary

Corollary 4.4. Let $\mu \in M_{\mathrm{s}, \mathrm{c}}^{+}$. Then $Z(b) \not \subset \mathcal{Z}(\mu)$ for every Blaschke product $b$.

Proof. Let $\left\{z_{n}\right\}_{n}$ be the zeros of $b$ in $D$. Then there is a subsequence $\left\{z_{n_{j}}\right\}_{j}$ such that $z_{n_{j}} \rightarrow \zeta$ for some $\zeta \in \partial D$. Let $b_{1}$ be the Blaschke product with zeros $\left\{z_{n_{j}}\right\}_{j}$. Then $S\left(b_{1}\right)=\{\zeta\}$. Hence by Corollary 4.3, $\mathcal{Z}(\mu) \cap Z\left(b_{1}\right)$ $=\emptyset$. Since $Z\left(b_{1}\right) \subset Z(b)$, we obtain our assertion.

Corollary 4.5. Let $\mu \in M_{\mathrm{s}, \mathrm{c}}^{+}$. Then $\operatorname{int} \mathcal{Z}(\mu)=\emptyset$.

Proof. Suppose that $\operatorname{int} \mathcal{Z}(\mu) \neq \emptyset$. Then there is an interpolating Blaschke product $b$ such that $Z(b) \subset \operatorname{int} \mathcal{Z}(\mu)$. But by Corollary $4.4, Z(b) \not \subset$ $\mathcal{Z}(\mu)$. This is a contradiction.

We have $\mathcal{W}(\mu) \cap M\left(L^{\infty}\right)=\emptyset$ for every $\mu \in M_{\mathrm{s}}^{+}$. Then by Corollary 3.8, for each $\zeta \in \partial D$ we have

$$
\mathcal{M}_{\zeta} \cap \overline{\bigcup_{\left\{\mu \in M_{\mathrm{s}}^{+} ; \mu(\{\zeta\})=0\right\}} \mathcal{W}(\mu)} \subset \mathcal{M}_{\zeta} \cap \overline{\bigcup_{\{\xi \in \partial D ; \xi \neq \zeta\}} \mathcal{M}_{\xi}} .
$$

Moreover we have the following.

Proposition 4.6. Let $\zeta \in \partial D$. Then

$$
\mathcal{M}_{\zeta} \cap \overline{\bigcup_{\left\{\mu \in M_{\mathrm{s}}^{+} ; \mu(\{\zeta\})=0\right\}} \mathcal{W}(\mu)} \varsubsetneqq \mathcal{M}_{\zeta} \cap \overline{\bigcup_{\{\xi \in \partial D ; \xi \neq \zeta\}} \mathcal{M}_{\xi}} .
$$

To prove this, we need a lemma.

Lemma 4.7. Let $\zeta \in \partial D$. Then there exists a sparse Blaschke product $b$ satisfying the following conditions.

(i) $S(b)=\{\zeta\}$.

(ii) Let $\mu \in M_{\mathrm{s}}^{+}$. Then there exists $\nu \in M_{\mathrm{S}}^{+}$such that $\nu \sim \mu$ and $\left|\psi_{\nu}\right|=1$ on $\overline{\{|b|<1\}}$. 
Proof. There exists a sequence $\left\{z_{n}\right\}_{n}$ in $D$ such that $\left|\psi_{\delta_{\zeta}}\left(z_{n}\right)\right| \rightarrow 1$ and $z_{n} \rightarrow \zeta$ as $n \rightarrow \infty$. Considering a subsequence, we may assume that $\left\{z_{n}\right\}_{n}$ is sparse. Let $b$ be the Blaschke product with zeros $\left\{z_{n}\right\}_{n}$. Then $S(b)=\{\zeta\}$, and by (4.2),

$$
\overline{\{|b|<1\}} \cap \overline{\bigcup_{\{\xi \in \partial D ; \xi \neq \zeta\}} \mathcal{M}_{\xi}}=\emptyset .
$$

Let $\mu \in M_{\mathrm{s}}^{+}$. Write $\mu=a \delta_{\zeta}+\mu_{1}$, where $\mu_{1}(\{\zeta\})=0$. Then by Proposition 3.7, there exists $\nu_{1} \in M_{\mathrm{s}}^{+}$such that $\nu_{1} \sim \mu_{1}$ and $\left|\psi_{\nu_{1}}\right|=1$ on $\overline{\{|b|<1\}}$. Since $Z(b)=\overline{\left\{z_{n}\right\}_{n}} \backslash\left\{z_{n}\right\}_{n}$, it follows that $\left|\psi_{\delta_{\zeta}}\right|=1$ on $Z(b)$. By Lemma 4.1, $\left|\psi_{\delta_{\zeta}}\right|=1$ on $\overline{\{|b|<1\}}$. Put $\nu=a \delta_{\zeta}+\nu_{1}$. Then $\nu \sim \mu$ and $\left|\psi_{\nu}\right|=\left|\psi_{\delta_{\zeta}}\right|^{a}\left|\psi_{\nu_{1}}\right|=1$ on $\overline{\{|b|<1\}}$.

Proof of Proposition 4.6. We may assume that $\zeta=1$. Let $\left\{J_{n}\right\}_{n}$ be a sequence of open subarcs of $\partial D$ such that $J_{n} \varsubsetneqq J_{n-1}$ and $\bigcap_{n=1}^{\infty} J_{n}=\{1\}$. Then there is a sequence $\left\{\xi_{n}\right\}_{n}$ such that $\xi_{n}$ is an interior point of $J_{n} \backslash J_{n-1}$ and $\xi_{n} \rightarrow 1$ as $n \rightarrow \infty$. We may assume that $\xi_{n} \neq \xi_{k}$ for $n \neq k$. Let $\mu \in M_{\mathrm{s}}^{+}$ and $\mu(\{1\})=0$. Put $\mu_{n}=\mu_{\mid\left(J_{n-1} \backslash J_{n}\right)}$. Then $\mu=\sum_{n=1}^{\infty} \mu_{n}$. For each $n$, by Lemma 4.7 there exist a sparse Blaschke product $q_{n}$ and $\nu_{n} \in M_{\mathrm{s}}^{+}$such that $S\left(q_{n}\right)=\left\{\xi_{n}\right\}, \nu_{n} \sim \mu_{n},\left\|\nu_{n}\right\|=\left\|\mu_{n}\right\|$, and $\left|\psi_{\nu_{n}}\right|=1$ on $Z\left(q_{n}\right)$. Let $\nu=\sum_{n=1}^{\infty} \nu_{n}$. Then $\nu \in M_{\mathrm{s}}^{+}$and $\nu \sim \mu$. Since $\xi_{n} \notin \operatorname{supp}\left(\nu-\nu_{n}\right)$, we have $\left|\psi_{\nu-\nu_{n}}\right|=1$ on $\mathcal{M}_{\xi_{n}}$. Since $S\left(q_{n}\right)=\left\{\xi_{n}\right\}$, it follows that $Z\left(q_{n}\right) \subset \mathcal{M}_{\xi_{n}}$. Hence

$$
\left|\psi_{\nu}\right|=\left|\psi_{\nu-\nu_{n}}\right|\left|\psi_{\nu_{n}}\right|=1 \quad \text { on } Z\left(q_{n}\right) .
$$

Let $\left\{w_{n, k}\right\}_{k}$ be the zeros of $q_{n}$. Then $w_{n, k} \rightarrow \xi_{n}$ as $k \rightarrow \infty$. Since $\xi_{n} \neq \xi_{k}$ for $n \neq k$, there is a sequence $\left\{N_{n}\right\}_{n}$ of positive integers such that $\left\{w_{n, k} ; k \geq N_{n}, n=1,2, \ldots\right\}$ is a sparse sequence (see [8, Lemma 1.5]). Since $\xi_{n} \rightarrow 1$, taking $N_{n}$ sufficiently large, we may assume that $\operatorname{cl}\left\{w_{n, k}\right.$; $\left.k \geq N_{n}\right\} \backslash\left\{w_{n, k} ; k \geq N_{n}\right\}=\{1\} \cup\left\{\xi_{n}\right\}_{n}$. Let $b$ be the associated sparse Blaschke product. Then $\bigcup_{n=1}^{\infty} Z\left(q_{n}\right) \subset Z(b)$ and $Z(b) \backslash \bigcup_{n=1}^{\infty} Z\left(q_{n}\right) \subset \mathcal{M}_{1}$. Hence by (4.4), $\left\{\left|\psi_{\nu}\right|<1\right\} \cap Z(b) \subset \mathcal{M}_{1}$.

For each positive integer $j$, let $b_{j}$ be a subproduct of $b$ with zeros

$$
\left\{w_{n, k} ;\left|\psi_{\nu}\left(w_{n, k}\right)\right|<1-1 / j, k \geq N_{n}, n=1,2, \ldots\right\} .
$$

Then $Z\left(b_{j}\right) \subset\left\{\left|\psi_{\nu}\right|<1\right\} \cap Z(b) \subset \mathcal{M}_{1}$. Hence

$$
Z\left(b_{j}\right) \cap \overline{\bigcup_{\{\xi \in \partial D ; \xi \neq 1\}} \mathcal{M}_{\xi}}=\emptyset .
$$

We also have

$$
\bigcup_{j=1}^{\infty} Z\left(b_{j}\right)=\left\{\left|\psi_{\nu}\right|<1\right\} \cap Z(b) .
$$


Therefore by Proposition 3.7 (considering $E=\{1\}$ ), there exists $\lambda \in M_{\mathrm{s}}^{+}$ such that $\lambda \sim \mu$ and

$$
\left|\psi_{\lambda}\right|=1 \quad \text { on }\left\{\left|\psi_{\nu}\right|<1\right\} \cap Z(b) .
$$

Let $\sigma=\nu \wedge \lambda$. Then $\sigma \sim \mu$ and $\left|\psi_{\sigma}\right| \geq \max \left\{\left|\psi_{\nu}\right|,\left|\psi_{\lambda}\right|\right\}$. Hence by (4.5), $\left|\psi_{\sigma}\right|=1$ on $Z(b)$. By Lemma 4.1, $\left\{\left|\psi_{\sigma}\right|<1\right\} \cap\{|b|<1\}=\emptyset$. Thus $\mathcal{W}(\mu) \cap$ $\{|b|<1\}=\emptyset$, so that

$$
\{|b|<1\} \cap \overline{\bigcup_{\left\{\mu \in M_{\mathrm{s}}^{+} ; \mu(\{1\})=0\right\}} \mathcal{W}(\mu)}=\emptyset .
$$

Since $\{|b|<1\} \cap \mathcal{M}_{\xi_{n}} \neq \emptyset$, it is not difficult to see that

$$
\{|b|<1\} \cap \mathcal{M}_{1} \cap \overline{\bigcup_{\{\xi \in \partial D ; \xi \neq 1\}} \mathcal{M}_{\xi}} \neq \emptyset .
$$

Thus we get our assertion.

By Lemma 4.7, we have the following.

Proposition 4.8. Let $\zeta \in \partial D$. Then there exists a Blaschke product $b$ such that $S(b)=\{\zeta\}$ and $\mathcal{Z}(\mu) \cap \overline{\{|b|<1\}}=\emptyset$ for every $\mu \in M_{\mathrm{s}}^{+}$.

One may ask whether there is a Blaschke product $b$ such that $S(b)=\partial D$ and $\mathcal{Z}(\mu) \cap \overline{\{|b|<1\}}=\emptyset$ for every $\mu \in M_{\mathrm{s}}^{+}$. The following says that the answer is "no".

TheOREM 4.9. Let $b$ be a Blaschke product such that $S(b)=\partial D$. Then

(i) $\mathcal{Z}\left(\delta_{\zeta}\right) \cap Z(b) \neq \emptyset$ for some $\zeta \in \partial D$.

(ii) $\mathcal{Z}(\mu) \cap Z(b) \neq \emptyset$ for some $\mu \in M_{\mathrm{s}, \mathrm{c}}^{+}$.

Proof. Let

$$
\Gamma\left(e^{i \theta}\right)=\left\{z \in D ; \frac{\left|e^{i \theta}-z\right|}{1-|z|}<2\right\} .
$$

Then

$$
\lim _{|z| \rightarrow 1, z \in \Gamma\left(e^{i \theta}\right)} \psi_{\delta_{e^{i \theta}}}(z)=0
$$

(see [5, p. 76]). Let $b$ be a Blaschke product such that $S(b)=\partial D$. Let $\left\{z_{n}\right\}_{n}$ be the zeros of $b$. Write

$$
z_{n}=r_{n} e^{i \theta_{n}} .
$$

By induction, we shall choose a subsequence $\left\{z_{n_{j}}\right\}_{j}$ of $\left\{z_{n}\right\}_{n}$. Put $n_{1}=1$. Since $S(b)=\partial D,\left\{e^{i \theta_{n}}\right\}_{n}$ is dense in $\partial D$. Then by (4.6), there exists a positive integer $n_{2}$ such that

$$
z_{n_{1}} \in \Gamma\left(e^{i \theta_{n_{2}}}\right), \quad \theta_{n_{1}}<\theta_{n_{2}}, \quad \theta_{n_{2}}-\theta_{n_{1}}<1 / 2 .
$$


Then $z_{n_{2}} \in \Gamma\left(e^{i \theta_{n_{2}}}\right)$, so that there exists $n_{3}$ such that

$$
z_{n_{1}}, z_{n_{2}} \in \Gamma\left(e^{i \theta_{n_{3}}}\right), \quad \theta_{n_{2}}<\theta_{n_{3}}, \quad \theta_{n_{3}}-\theta_{n_{2}}<1 / 2^{2} .
$$

Continuing, we get $\left\{z_{n_{j}}\right\}_{j}$ satisfying

$$
z_{n_{k}} \in \Gamma\left(e^{i \theta_{n_{j}}}\right) \quad \text { for } 1 \leq k \leq j, \quad \theta_{n_{j}}<\theta_{n_{j+1}} \quad \theta_{n_{j+1}}-\theta_{n_{j}}<1 / 2^{j+1} .
$$

Thus $\theta_{n_{j}} \rightarrow \theta_{0}$ as $j \rightarrow \infty$ for some $\theta_{0}$. By (4.8), $z_{n_{k}} \in \operatorname{cl} \Gamma\left(e^{i \theta_{0}}\right)$ for every $k$. Then by (4.7), $\psi_{\delta_{e^{i \theta_{0}}}}\left(z_{n_{k}}\right) \rightarrow 0$ as $k \rightarrow \infty$, so that $Z\left(\psi_{\delta_{e^{i \theta_{0}}}}\right) \cap Z(b) \neq \emptyset$. Therefore we get $\mathcal{Z}\left(\delta_{e^{i \theta_{0}}}\right) \cap Z(b) \neq \emptyset$.

To prove (ii), we need to work more. In the proof of (i), we choose one point in each step. In the proof of (ii), we choose two points. Let

$$
\Lambda_{k}=\left\{\left(\alpha_{1}, \ldots, \alpha_{k}\right) ; \alpha_{j}=0 \text { or } 1\right\}, \quad \Lambda_{\infty}=\left\{\left(\alpha_{1}, \alpha_{2}, \ldots\right) ; \alpha_{j}=0 \text { or } 1\right\} .
$$

For $\alpha=\left(\alpha_{1}, \ldots, \alpha_{k}\right) \in \Lambda_{k}$, put $|\alpha|=k$ and $\alpha^{j}=\left(\alpha_{1}, \ldots, \alpha_{j}\right)$ for $j \leq k$. By induction, we shall choose a sequence $\left\{n_{\alpha} ; \alpha \in \Lambda_{k}\right\}, k=1,2, \ldots$, of finite sets of positive integers. Take positive integers $n_{0}$ and $n_{1}$ such that $\theta_{n_{0}}<\theta_{n_{1}}$. We have

$$
z_{n_{0}} \in \Gamma\left(e^{i \theta_{n_{0}}}\right), \quad z_{n_{1}} \in \Gamma\left(e^{i \theta_{n_{1}}}\right) .
$$

Then take $n_{(l, m)}$ for $l, m=0,1$ such that

$$
\begin{gathered}
z_{n_{l}} \in \Gamma\left(e^{i \theta_{n_{(l, m)}}}\right) \quad \text { for } l, m=0,1, \\
0<\left|\theta_{n_{(l, m)}}-\theta_{n_{l}}\right|<\left|\theta_{n_{1}}-\theta_{n_{0}}\right| / 4 \quad \text { for } l, m=0,1, \\
\theta_{n_{(l, m)}} \neq \theta_{n_{(t, s)}} \quad \text { if }(l, m) \neq(t, s) .
\end{gathered}
$$

Assume that $\left\{n_{\alpha} ; \alpha \in \Lambda_{j}\right\}, 1 \leq j \leq k$, are chosen so that $z_{n_{\alpha j}} \in \Gamma\left(e^{i \theta_{n_{\alpha}}}\right)$ for $1 \leq j \leq|\alpha|$ and $\theta_{n_{\alpha}} \neq \theta_{n_{\beta}}$ for $\alpha, \beta \in \bigcup_{j=1}^{k} \Lambda_{j}, \alpha \neq \beta$. Let $\alpha \in \Lambda_{k}$. Take $n_{(\alpha, 0)}$ and $n_{(\alpha, 1)}$ such that

$$
\begin{gathered}
z_{n_{\alpha} j} \in \Gamma\left(e^{i \theta_{n}(\alpha, l)}\right) \quad \text { for } 1 \leq j \leq k \text { and } l=0,1, \\
0<\left|\theta_{n_{(\alpha, l)}}-\theta_{n_{\alpha}}\right| \\
<\frac{1}{4} \min \left\{\left|\theta_{n_{\lambda}}-\theta_{n_{\gamma}}\right| ; \lambda, \gamma \in \bigcup_{j=1}^{k} \Lambda_{j}, \lambda \neq \gamma\right\} \quad \text { for } l=0,1 .
\end{gathered}
$$

This finishes our induction.

Let $\alpha=\left(\alpha_{1}, \alpha_{2}, \ldots\right) \in \Lambda_{\infty}$. Put $\alpha^{k}=\left(\alpha_{1}, \ldots, \alpha_{k}\right) \in \Lambda_{k}$. Then by (4.10),

$$
\left|\theta_{n_{\alpha^{k}}}-\theta_{n_{\alpha^{j}}}\right|<\left(\frac{1}{4}\right)^{k-1}\left(\sum_{l=1}^{j-k}\left(\frac{1}{4}\right)^{l}\right)\left|\theta_{n_{1}}-\theta_{n_{0}}\right| \quad \text { for } j>k .
$$

Hence $\left\{\theta_{n_{\alpha^{k}}}\right\}_{k}$ converges to some point, say $\theta_{\alpha}$. By (4.9),

$$
z_{n_{\alpha j}} \in \Gamma\left(e^{i \theta_{\alpha}}\right) \quad \text { for every } j \text {. }
$$


Let $\beta \in \Lambda_{\infty}$ and $\alpha \neq \beta$. Then we may assume that

$$
\alpha=\left(\alpha_{1}, \ldots, \alpha_{k}, 0, \alpha_{k+2}, \ldots\right), \quad \beta=\left(\alpha_{1}, \ldots, \alpha_{k}, 1, \beta_{k+2}, \ldots\right) .
$$

By (4.10), we have

$$
\left|\theta_{n_{\alpha} j}-\theta_{n_{\left(\alpha_{1}, \ldots, \alpha_{k}, 0\right)}}\right|<\sum_{l=1}^{j-k-1}\left(\frac{1}{4}\right)^{l}\left|\theta_{n_{\left(\alpha_{1}, \ldots, \alpha_{k}, 0\right)}}-\theta_{n_{\left(\alpha_{1}, \ldots, \alpha_{k}, 1\right)}}\right| \quad \text { for } j \geq k+2 .
$$

Hence

$$
\left|\theta_{\alpha}-\theta_{n_{\left(\alpha_{1}, \ldots, \alpha_{k}, 0\right)}}\right|<\frac{1}{3}\left|\theta_{n_{\left(\alpha_{1}, \ldots, \alpha_{k}, 0\right)}}-\theta_{n_{\left(\alpha_{1}, \ldots, \alpha_{k}, 1\right)}}\right| .
$$

Similarly,

$$
\left|\theta_{\beta}-\theta_{n_{\left(\alpha_{1}, \ldots, \alpha_{k}, 1\right)}}\right|<\frac{1}{3}\left|\theta_{n_{\left(\alpha_{1}, \ldots, \alpha_{k}, 0\right)}}-\theta_{n_{\left(\alpha_{1}, \ldots, \alpha_{k}, 1\right)}}\right| .
$$

Thus we get $\theta_{\alpha} \neq \theta_{\beta}$. By our construction, $\left\{\theta_{\alpha} ; \alpha \in \Lambda_{\infty}\right\}$ is the set of cluster points of $\bigcup_{k=1}^{\infty}\left\{\theta_{n_{\alpha}} ; \alpha \in \Lambda_{k}\right\}$. Hence $\left\{\theta_{\alpha} ; \alpha \in \Lambda_{\infty}\right\}$ is a perfect set. Then there exists $\mu \in M_{\mathrm{s}, \mathrm{c}}^{+}$such that $\operatorname{supp} \mu \subset\left\{\theta_{\alpha} ; \alpha \in \Lambda_{\infty}\right\}$. By [5, p. 76],

$$
\lim _{|z| \rightarrow 1, z \in \Gamma\left(\theta_{\alpha}\right)} \psi_{\mu}(z)=0
$$

for some $\alpha \in \Lambda_{\infty}$. Therefore by (4.11), we have $Z\left(\psi_{\mu}\right) \cap Z(b) \neq \emptyset$. By Lemma 3.2 , we obtain $\mathcal{Z}(\mu) \cap Z(b) \neq \emptyset$.

Here we have the following problem.

Problem 4.10. Does there exist an interpolating Blaschke product $b_{0}$ such that $S\left(b_{0}\right)=\partial D$ and $\mathcal{Z}(\mu) \cap Z\left(b_{0}\right) \neq \emptyset$ for every $\mu \in M_{\mathrm{s}}^{+}$?

5. Construction of interpolating Blaschke products. For a measurable subset $E$ of $\partial D$, we denote by $|E|$ the Lebesgue measure of $E$. In this section, for a given closed subset $K$ of $\partial D$ with $|K|=0$, we construct a special interpolating Blaschke product $b_{K}$ associated with $K$. In Section 6 , we shall prove that $Z\left(b_{K}\right) \cap \mathcal{Z}(\mu) \neq \emptyset$ for every $\mu \in M_{\mathrm{s}}^{+}$with $\operatorname{supp} \mu \subset K$.

TheOREm 5.1. Let $K$ be a closed subset of $\partial D$ with $|K|=0$. Then there exists a sequence $\left\{J_{n, j}\right\}_{j=1}^{N_{n}}, n=1,2, \ldots$, of open arcs such that for every $n$ and $k$,

$$
\begin{gathered}
K \subset \bigcup_{j=1}^{N_{n}} J_{n, j} \subset \bigcup_{j=1}^{N_{n-1}} J_{n-1, j}, \\
\sum_{j}\left\{\left|J_{n, j}\right| ; J_{n, j} \subset J_{n-1, k}\right\} \leq\left|J_{n-1, k}\right| / 2 .
\end{gathered}
$$


Let $e^{i \theta_{n, j}}$ be the center of the arc $J_{n, j}$ and

$$
z_{n, j}=\left(1-\frac{\left|J_{n, j}\right|}{2 \pi}\right) e^{i \theta_{n, j}}
$$

Then $\left\{z_{n, j} ; 1 \leq j \leq N_{n}, n=1,2, \ldots\right\}$ is an interpolating sequence and the set of cluster points of $\left\{z_{n, j} ; 1 \leq j \leq N_{n}, n=1,2, \ldots\right\}$ in the closed unit disk $\bar{D}$ coincides with $K$.

Let $b_{K}$ be the Blaschke product with zeros $\left\{z_{n, j} ; 1 \leq j \leq N_{n}, n=\right.$ $1,2, \ldots\}$. We call $b_{K}$ the interpolating Blaschke product associated with $K$.

Proof of Theorem 5.1. Let $K$ be a non-empty closed subset of $\partial D$ and $|K|=0$. Then $K$ is totally disconnected. For an open arc $V$ of $\partial D$ such that $V \cap K$ is a non-empty closed set, there are finitely many disjoint open arcs $\left\{V_{j}\right\}_{j=1}^{k}$ of $\partial D$ such that $V_{j} \cap K$ are non-empty closed sets and

$$
V \cap K \subset \bigcup_{j=1}^{k} V_{j} \subset V, \quad \sum_{j=1}^{k}\left|V_{j}\right| \leq|V| / 2 .
$$

Now using the above fact inductively, we shall choose a family $\left\{J_{n, j}\right\}_{j=1}^{N_{n}}$ of open arcs for each $n$. Let $J_{0}=\partial D$. Put $V=J_{0}$ in the above; then there are finitely many disjoint open arcs $\left\{J_{1, j}\right\}_{j=1}^{N_{1}}$ of $\partial D$ such that $J_{1, j} \cap K$ are non-empty closed sets and

$$
J_{0} \cap K \subset \bigcup_{j=1}^{N_{1}} J_{1, j} \subset J_{0}, \quad \sum_{j=1}^{N_{1}}\left|J_{1, j}\right| \leq\left|J_{0}\right| / 2 .
$$

We proceed to the next step. For each $J_{1, j}, 1 \leq j \leq N_{1}$, there are finitely many disjoint open arcs $\left\{J_{1, j, l}\right\}_{l=1}^{m_{j}}$ of $\partial D$ such that $J_{1, j, l} \cap K$ are non-empty closed sets and

$$
J_{1, j} \cap K \subset \bigcup_{l=1}^{m_{j}} J_{1, j, l} \subset J_{1, j}, \quad \sum_{l=1}^{m_{j}}\left|J_{1, j, l}\right| \leq\left|J_{1, j}\right| / 2 .
$$

Let $N_{2}=\sum_{j=1}^{N_{1}} m_{j}$ and

$$
\left\{J_{2, j}\right\}_{j=1}^{N_{2}}=\left\{J_{1, j, l} ; 1 \leq j \leq N_{1}, 1 \leq l \leq m_{j}\right\}
$$

We have

$$
K \subset \bigcup_{j=1}^{N_{2}} J_{2, j}
$$

Continuing this process, at the $n$th step we have a finite family $\left\{J_{n, j}\right\}_{j=1}^{N_{n}}$ of disjoint open arcs of $\partial D$ such that for $1 \leq k \leq N_{n-1}$,

$$
J_{n-1, k} \cap K \subset \bigcup_{j}\left\{J_{n, j} ; J_{n, j} \subset J_{n-1, k}\right\} \subset J_{n-1, k},
$$


$J_{n, j} \cap K$ is non-empty closed for every $j$ with $1 \leq j \leq N_{n}$,

$$
\begin{gathered}
K \subset \bigcup_{j=1}^{N_{n}} J_{n, j} \subset \bigcup_{j=1}^{N_{n-1}} J_{n-1, j}, \\
\sum_{j}\left\{\left|J_{n, j}\right| ; J_{n, j} \subset J_{n-1, k}\right\} \leq\left|J_{n-1, k}\right| / 2 .
\end{gathered}
$$

Thus we get the first half of our assertion.

By the above, we have

$$
\bigcap_{n=1}^{\infty} \bigcup_{j=1}^{N_{n}} J_{n, j}=K
$$

Let $1 \leq j \leq N_{n}$. For $l>n$, we have

$$
\begin{aligned}
\sum_{t}\left\{\left|J_{l, t}\right| ; J_{l, t} \subset J_{n, j}\right\} & =\sum_{k} \sum_{t}\left\{\left|J_{l, t}\right| ; J_{l, t} \subset J_{l-1, k} \subset J_{n, j}\right\} & & \text { by (5.1) } \\
& =\frac{1}{2} \sum_{k}\left\{\left|J_{l-1, k}\right| ; J_{l-1, k} \subset J_{n, j}\right\} & & \text { by (5.3). }
\end{aligned}
$$

Hence

$$
\sum_{t}\left\{\left|J_{l, t}\right| ; J_{l, t} \subset J_{n, j}\right\} \leq\left(\frac{1}{2}\right)^{l-n}\left|J_{n, j}\right|,
$$

so that

$$
\sum_{l=n}^{\infty} \sum_{t}\left\{\left|J_{l, t}\right| ; J_{l, t} \subset J_{n, j}\right\} \leq 2\left|J_{n, j}\right| .
$$

For $n \geq 1$ and $1 \leq j \leq N_{n}$, let $e^{i \theta_{n, j}}$ be the center of the arc $J_{n, j}$,

$$
z_{n, j}=\left(1-\frac{\left|J_{n, j}\right|}{2 \pi}\right) e^{i \theta_{n, j}}
$$

and

$$
R\left(z_{n, j}\right)=\left\{r e^{i \theta} ; e^{i \theta} \in J_{n, j}, 1-\left|J_{n, j}\right| / 2 \pi \leq r<1\right\} .
$$

Then $z_{n, j} \in R\left(z_{n, j}\right)$ and $1-\left|z_{n, j}\right|=\left|J_{n, j}\right| / 2 \pi$. By (5.4), $K$ is the set of cluster points of $\left\{z_{n, j} ; 1 \leq j \leq N_{n}, n=1,2, \ldots\right\}$ in $\bar{D}$.

We prove that $\left\{z_{n, j} ; 1 \leq j \leq N_{n}, n=1,2, \ldots\right\}$ is an interpolating sequence. It is not difficult to see that $\left\{z_{n, j}\right\}_{n, j}$ is $\varrho$-separated, that is,

$$
\inf \left\{\varrho\left(z_{n, j}, z_{k, l}\right) ;(n, j) \neq(k, l)\right\}>0 ;
$$

I leave the proof to the reader. To prove that $\left\{z_{n, j} ; 1 \leq j \leq N_{n}, n=1,2, \ldots\right\}$ 
is interpolating, it is sufficient to show that

$$
\sigma=\sum_{n=1}^{\infty} \sum_{j=1}^{N_{n}}\left(1-\left|z_{n, j}\right|\right) \delta_{z_{n, j}}=\sum_{n=1}^{\infty} \sum_{j=1}^{N_{n}}\left|J_{n, j}\right| \delta_{z_{n, j}} / 2 \pi
$$

is a Carleson measure (see [2] and also [5, pp. 286-287]). Let

$$
\Omega=\left\{r e^{i \theta} ; 1-\varepsilon \leq r<1, \theta_{0} \leq \theta \leq \theta_{0}+2 \pi \varepsilon\right\}, \quad \text { where } 0<\varepsilon<1,
$$

be an arbitrary Carleson square. We need to show that there is an absolute constant $C$, independent of $\varepsilon$, such that

$$
\sum_{n, j}\left\{\left|J_{n, j}\right| ; z_{n, j} \in \Omega\right\} \leq C \varepsilon .
$$

By our construction, there exists a sequence $\left\{z_{n_{k}, j_{k}}\right\}_{k=1}^{\infty}$ (maybe a finite set) satisfying

$$
\begin{gathered}
z_{n_{k}, j_{k}} \in \Omega \quad \text { for every } k, \\
R\left(z_{n_{k}, j_{k}}\right) \cap R\left(z_{n_{i}, j_{l}}\right)=\emptyset \quad \text { for every } k \neq l,
\end{gathered}
$$

(5.11) if $z_{n, j} \in \Omega$, there exists $k$ such that $R\left(z_{n, j}\right) \subset R\left(z_{n_{k}, j_{k}}\right)$.

Then

$$
\begin{aligned}
\sum_{n, j}\left\{\left|J_{n, j}\right| ; z_{n, j} \in \Omega\right\} & =\sum_{k=1}^{\infty}\left(\sum_{n, j}\left\{\left|J_{n, j}\right| ; R\left(z_{n, j}\right) \subset R\left(z_{n_{k}, j_{k}}\right)\right\}\right) \\
& =\sum_{k=1}^{\infty}\left(\sum_{n, j}\left\{\left|J_{n, j}\right| ; J_{n, j} \subset J_{n_{k}, j_{k}}\right\}\right) \quad \text { by }(5.6) \\
& \leq 2 \sum_{k=1}^{\infty}\left|J_{n_{k}, j_{k}}\right| \quad \text { by }(5.5) .
\end{aligned}
$$

By (5.6) and (5.10), $J_{n_{k}, j_{k}} \cap J_{n_{m}, j_{m}}=\emptyset$ if $k \neq m$. By (5.9),

$$
J_{n_{k}, j_{k}} \cap\left\{e^{i \theta} ; \theta_{0} \leq \theta \leq \theta_{0}+2 \pi \varepsilon\right\} \neq \emptyset
$$

and

$$
\left|\left\{e^{i \theta} ; \theta_{0} \leq \theta \leq \theta_{0}+2 \pi \varepsilon\right\}\right| \geq\left|J_{n_{k}, j_{k}}\right| .
$$

Hence by (5.7),

$$
\sum_{k=1}^{\infty}\left|J_{n_{k}, j_{k}}\right| \leq 6 \pi \varepsilon
$$

Thus we get (5.8), so that $\left\{z_{n, j} ; 1 \leq j \leq N_{n}, n=1,2, \ldots\right\}$ is interpolating. This completes the proof. 
6. Properties of $\mathcal{Z}(\mu)$. First we prove the following theorem.

THEOREM 6.1. Let $K$ be a non-empty closed subset of $\partial D$ with $|K|=0$, and $\mu \in M_{\mathrm{s}}^{+}$be such that $\operatorname{supp} \mu \subset K$. Then $Z\left(b_{K}\right) \cap \mathcal{Z}(\mu) \neq \emptyset$, where $b_{K}$ is the interpolating Blaschke product associated with $K$.

Let $K$ be a non-empty closed subset of $\partial D$ with $|K|=0$. Generally, there are uncountably many measures $\left\{\mu_{\alpha}\right\}_{\alpha \in \Lambda}$ in $M_{\mathrm{s}}^{+}$such that supp $\mu_{\alpha} \subset K$ and $\mu_{\alpha} \perp \mu_{\beta}$ if $\alpha \neq \beta$. By Theorems 3.1 and $6.1,\left\{Z\left(b_{K}\right) \cap \mathcal{Z}\left(\mu_{\alpha}\right)\right\}_{\alpha}$ is a family of non-empty mutually disjoint subsets in $Z\left(b_{K}\right)$. So $b_{K}$ is a very convenient interpolating Blaschke product to study the properties of $\psi_{\mu}$ with $\operatorname{supp} \mu \subset K$.

Proof of Theorem 6.1. Let $\nu \in M_{\mathrm{s}}^{+}$and $\nu \sim \mu$. We show that

$$
Z\left(b_{K}\right) \cap Z\left(\psi_{\nu}\right) \neq \emptyset \text {. }
$$

Let $\left\{J_{n, j} ; 1 \leq j \leq N_{n}, n=1,2, \ldots\right\}$ and $\left\{z_{n, j} ; 1 \leq j \leq N_{n}, n=1,2, \ldots\right\}$ be families given in Theorem 5.1. First, we prove that

$$
\limsup _{n \rightarrow \infty} \max _{1 \leq j \leq N_{n}} \frac{\nu\left(J_{n, j}\right)}{\left|J_{n, j}\right|}=\infty .
$$

Suppose not. Then there exists a positive constant $C$ such that

$$
\max _{1 \leq j \leq N_{n}} \frac{\nu\left(J_{n, j}\right)}{\left|J_{n, j}\right|} \leq C \quad \text { for every } n .
$$

Then for each $n$, we have

$$
\begin{array}{rlr}
\nu(K) & \leq \sum_{j=1}^{N_{n}} \nu\left(J_{n, j}\right) \quad \text { by Theorem 5.1(i) } \\
& \leq C \sum_{j=1}^{N_{n}}\left|J_{n, j}\right| \quad \text { by }(6.3) \\
& \leq \frac{C}{2} \sum_{j=1}^{N_{n-1}}\left|J_{n-1, j}\right| \quad \text { by Theorem 5.1(ii) } \\
& \leq \frac{2 \pi C}{2^{n}} .
\end{array}
$$

Hence $\nu(K)=0$, contrary to our assumption, so that (6.2) holds.

By (6.2), there exist $\left\{n_{k}\right\}_{k}$ and $\left\{j_{k}\right\}_{k}$ such that $1 \leq j_{k} \leq N_{n_{k}}$ and

$$
\frac{\nu\left(J_{n_{k}, j_{k}}\right)}{\left|J_{n_{k}, j_{k}}\right|} \rightarrow \infty \quad \text { as } k \rightarrow \infty \text {. }
$$

By Theorem 5.1,

$$
\left|J_{n_{k}, j_{k}}\right|=2 \pi\left(1-\left|z_{n_{k}, j_{k}}\right|\right)
$$


Let $e^{i t} \in J_{n_{k}, j_{k}}$. Then

$$
\begin{aligned}
\left|e^{i t}-z_{n_{k}, j_{k}}\right| & \leq|| z_{n_{k}, j_{k}} \mid-e^{i \pi\left(1-\left|z_{n_{k}, j_{k}}\right|\right) \mid} \\
& \leq\left(1-\left|z_{n_{k}, j_{k}}\right|\right)+\left|1-e^{i \pi\left(1-\left|z_{n_{k}, j_{k}}\right|\right)}\right| \\
& \leq(1+\pi)\left(1-\left|z_{n_{k}, j_{k}}\right|\right) .
\end{aligned}
$$

Then

$$
\left|P_{z_{n_{k}, j_{k}}}\left(e^{i t}\right)\right|=\frac{1-\left|z_{n_{k}, j_{k}}\right|^{2}}{\left|e^{i t}-z_{n_{k}, j_{k}}\right|^{2}} \geq \frac{1}{(1+\pi)^{2}\left(1-\left|z_{n_{k}, j_{k}}\right|\right)} .
$$

Hence by (6.5),

$$
\left|P_{z_{n_{k}, j_{k}}}\right| \geq \frac{2 \pi}{(1+\pi)^{2}\left|J_{n_{k}, j_{k}}\right|} \quad \text { on } J_{n_{k}, j_{k}} .
$$

Consequently, we have

$$
\begin{aligned}
-\log \left|\psi_{\nu}\left(z_{n_{k}, j_{k}}\right)\right| & =\int_{0}^{2 \pi} P_{z_{n_{k}, j_{k}}}\left(e^{i \theta}\right) d \nu(\theta) \geq \int_{J_{n_{k}, j_{k}}} P_{z_{n_{k}, j_{k}}}\left(e^{i \theta}\right) d \nu(\theta) \\
& \geq \frac{2 \pi \nu\left(J_{n_{k}, j_{k}}\right)}{(1+\pi)^{2}\left|J_{n_{k}, j_{k}}\right|} .
\end{aligned}
$$

Therefore by (6.4), $\psi_{\nu}\left(z_{n_{k}, j_{k}}\right) \rightarrow 0$ as $k \rightarrow \infty$. Since $b_{K}$ is the Blaschke product with zeros $\left\{z_{n, j} ; 1 \leq j \leq N_{n}, n=1,2, \ldots\right\}$, we obtain $Z\left(b_{K}\right) \cap$ $Z\left(\psi_{\nu}\right) \neq \emptyset$. Then Lemma 3.2 yields the assertion.

Corollary 6.2. Let $\mu \in M_{\mathrm{s}}^{+}$. Then $\mathcal{Z}(\mu)$ contains non-trivial points.

Proof. Since $\mu$ is a singular measure, there exists a closed subset $K$ of $\partial D$ such that $|K|=0$ and $\mu(K)>0$. By Lemma 3.3(ii), $\mathcal{Z}\left(\mu_{\mid K}\right) \subset \mathcal{Z}(\mu)$, and by Theorem $6.1, \emptyset \neq Z\left(b_{K}\right) \cap \mathcal{Z}\left(\mu_{\mid K}\right) \subset Z\left(b_{K}\right) \cap \mathcal{Z}(\mu)$. Since $b_{K}$ is interpolating, we have $Z\left(b_{K}\right) \subset G$.

Let $\mu \in M_{\mathrm{s}}^{+}$. We denote by $M\left(L^{\infty}(\mu)\right)$ the maximal ideal space of the Banach algebra $L^{\infty}(\mu)$. Then $M\left(L^{\infty}(\mu)\right)$ is a totally disconnected space. For $f \in L^{\infty}(\mu)$, let $\widehat{f}$ be the Gelfand transform of $f$. For a measurable subset $S$ of supp $\mu$, there exists an open and closed subset $\widehat{S}$ of $M\left(L^{\infty}(\mu)\right)$ such that $\widehat{\chi}_{S}=\chi_{\widehat{S}}$. Then the family $\left\{\chi_{\widehat{S}}\right\}_{S}$ coincides with the set of idempotents in $C\left(M\left(L^{\infty}(\mu)\right)\right)$, the space of continuous functions on $M\left(L^{\infty}(\mu)\right)$. We have $\widehat{S^{\mathrm{c}}}=(\widehat{S})^{\mathrm{c}}$. For each $x \in M\left(L^{\infty}(\mu)\right)$, let

$$
\Phi_{\mu}(x)=\bigcap_{\{S ; x \in \widehat{S}\}} \mathcal{Z}\left(\mu_{\mid S}\right) .
$$

The set $\Phi_{\mu}(x)$ is a closed subset in $\mathcal{M}$ associated with the point $x \in$ $M\left(L^{\infty}(\mu)\right)$. It is interesting to study $\Phi_{\mu}(x)$ from the point of view of measures on $\partial D$.

We have the following. 
Theorem 6.3. Let $\mu \in M_{\mathrm{s}}^{+}$.

(i) $\emptyset \neq \Phi_{\mu}(x) \subset \mathcal{Z}(\mu)$ for $x \in M\left(L^{\infty}(\mu)\right)$.

(ii) $\Phi_{\mu}(x) \cap \Phi_{\mu}(y)=\emptyset$ if $x, y \in M\left(L^{\infty}(\mu)\right)$ and $x \neq y$.

(iii) $\mathcal{Z}(\mu)=\bigcup_{x \in M\left(L^{\infty}(\mu)\right)} \Phi_{\mu}(x)$.

Proof. First, assume that $\mu=\delta_{\zeta}$ for some $\zeta \in \partial D$. Then $M\left(L^{\infty}(\mu)\right)$ is a one-point set, say $\{x\}$, and it is easy to see that $\Phi_{\mu}(x)=Z\left(\psi_{\delta_{\zeta}}\right)=\mathcal{Z}\left(\delta_{\zeta}\right)$. Hence we obtain the assertion.

Next suppose that $\mu$ is not a point mass. Then $M\left(L^{\infty}(\mu)\right)$ contains more than one point. Let $S$ be a measurable subset of $\operatorname{supp} \mu$. Then $\mu=\mu_{\mid S}+\mu_{\mid S^{c}}$ and $\mu_{\mid S} \perp \mu_{\mid S^{c}}$. Hence by Theorem $2.2, \mathcal{Z}\left(\mu_{\mid S}\right) \cap \mathcal{Z}\left(\mu_{\mid S^{c}}\right)=\emptyset$ and $\mathcal{Z}\left(\mu_{\mid S}\right) \subset$ $\mathcal{Z}(\mu)$. By Lemma 3.3, $\mathcal{Z}(\mu)=\mathcal{Z}\left(\mu_{\mid S}\right) \cup \mathcal{Z}\left(\mu_{\mid S^{c}}\right)$. Thus if $\mu_{\mid S} \neq 0$, then $\mathcal{Z}\left(\mu_{\mid S}\right)$ is a non-empty open and closed subset of $\mathcal{Z}(\mu)$.

Let $x \in M\left(L^{\infty}(\mu)\right)$. Suppose that $\Phi_{\mu}(x)=\emptyset$. Then there exist $S_{1}, \ldots, S_{n}$ such that $x \in \widehat{S}_{j}$ for every $j$ and $\bigcap_{j=1}^{n} \mathcal{Z}\left(\mu_{\mid S_{j}}\right)=\emptyset$. Set $S=\bigcap_{j=1}^{n} S_{j}$. Then $x \in \widehat{S}$, so that $\mu_{\mid S} \neq 0$. Hence by Proposition 3.1, $\mathcal{Z}\left(\mu_{\mid S}\right) \neq \emptyset$. By Lemma 3.3, $\mathcal{Z}\left(\mu_{\mid S}\right) \subset \bigcap_{j=1}^{n} \mathcal{Z}\left(\mu_{\mid S_{j}}\right)$. This is a contradiction. Thus we get (i).

Let $x, y \in M\left(L^{\infty}(\mu)\right)$ and $x \neq y$. Then there exists $S$ such that $x \in \widehat{S}$ and $y \notin \widehat{S}$. We have $y \in \widehat{S^{\mathrm{c}}}$, and hence by Theorem 2.2 ,

$$
\Phi_{\mu}(x) \cap \Phi_{\mu}(y) \subset \mathcal{Z}\left(\mu_{\mid S}\right) \cap \mathcal{Z}\left(\mu_{\mid S^{c}}\right)=\emptyset .
$$

Thus (ii) holds.

Suppose (iii) does not hold. Then there is $\zeta \in \mathcal{Z}(\mu)$ such that $\zeta \notin$ $\Phi_{\mu}(x)$ for every $x \in M\left(L^{\infty}(\mu)\right)$. Hence for each $x \in M\left(L^{\infty}\right)$, there exists a measurable subset $S_{x}$ of supp $\mu$ such that $x \in \widehat{S}_{x}$ and $\zeta \notin \mathcal{Z}\left(\mu_{\mid S_{x}}\right)$. Since $\widehat{S}_{x}$ is an open subset of $M\left(L^{\infty}(\mu)\right)$, there exist $S_{x_{1}}, \ldots, S_{x_{n}}$ such that

$$
M\left(L^{\infty}(\mu)\right)=\bigcup_{j=1}^{n} \widehat{S}_{x_{j}} .
$$

Put $S=\bigcup_{j=1}^{n} S_{x_{j}}$. Then $\widehat{S}=\bigcup_{j=1}^{n} \widehat{S}_{x_{j}}=M\left(L^{\infty}(\mu)\right)$, so that $\mu_{\mid S}=\mu$. By Lemma 3.3,

$$
\mathcal{Z}(\mu)=\bigcup_{j=1}^{n} \mathcal{Z}\left(\mu_{\mid S_{x_{j}}}\right) .
$$

Hence $\zeta \in \mathcal{Z}\left(\mu_{\mid S_{x_{j}}}\right)$ for some $j$. This is a contradiction.

We have the following problem.

Problem 6.4. Let $\mu \in M_{\mathrm{S}}^{+}$. Is $\Phi_{\mu}(x)$ a connected set for every $x \in$ $M\left(L^{\infty}(\mu)\right) ?$

We give some results on the sets $\Phi_{\mu}(x)$. 
Proposition 6.5. Let $\mu \in M_{\mathrm{s}}^{+}$and $x \in M\left(L^{\infty}(\mu)\right)$.

(i) If $\zeta \in \Phi_{\mu}(x)$, then $P(\zeta) \subset \Phi_{\mu}(x)$.

(ii) $\Phi_{\mu}(x)$ contains trivial points.

(iii) If $\mu \in M_{\mathrm{s}, \mathrm{c}}^{+}, \zeta \in \Phi_{\mu}(x)$, supp $\mu_{\zeta} \subset \operatorname{supp} \mu_{\xi}$, and $\xi \in \mathcal{M} \backslash D$, then $\xi \in \Phi_{\mu}(x)$.

Proof. Let $\zeta \in \Phi_{\mu}(x)$. Then $\psi_{\nu}(\zeta)=0$ for every $\nu \in M_{\mathrm{s}}^{+}$with $\nu \sim \mu$. Since $\psi_{\nu}$ is a singular inner function, we have $P(\zeta) \subset Z\left(\psi_{\nu}\right)$. Hence $P(\zeta) \subset$ $\mathcal{Z}(\mu)$. Thus we get (i).

(ii) follows from (i) and Budde's theorem [1], and (iii) from Corollary 3.10 and (6.6).

One may ask whether each $\Phi_{\mu}(x)$ contains non-trivial points. Here is the answer.

Theorem 6.6. Let $\mu \in M_{\mathrm{s}}^{+}$and $x \in M\left(L^{\infty}(\mu)\right)$. Then $\Phi_{\mu}(x)$ contains non-trivial points.

Proof. Let $\mu \in M_{\mathrm{s}}^{+}$. By the regularity of $\mu$, there is a sequence $\left\{K_{n}\right\}_{n}$ (maybe finite) of non-empty closed subsets satisfying

$$
\begin{gathered}
\left|K_{n}\right|=0 \quad \text { for every } n, \\
K_{n} \cap K_{m}=\emptyset \quad \text { if } n \neq m, \\
\mu=\sum_{n=1}^{\infty} \mu_{\mid K_{n}} .
\end{gathered}
$$

For each $n$, there exists an interpolating Blaschke product $b_{K_{n}}$ associated with $K_{n}$. Let $\left\{w_{n, j}\right\}_{j}$ be the zeros of $b_{K_{n}}$ in $D$. Then by Theorem 5.1, $K_{n}$ is the set of cluster points of $\left\{w_{n, j}\right\}_{j}$ in $\bar{D}$. Then by (6.8), we have $\left\{\left|b_{K_{n}}\right|<1\right\} \cap\left\{\left|b_{K_{m}}\right|<1\right\}=\emptyset$ if $n \neq m$. By the proof of [8, Lemma 1.5], there is a sequence $\left\{k_{j}\right\}_{j}$ of positive integers such that $\left\{w_{n, j} ; j \geq k_{n}, n=1,2, \ldots\right\}$ is an interpolating sequence.

Let

$$
b_{K_{n}}^{\prime}(z)=\prod_{j=k_{n}}^{\infty} \frac{-\bar{w}_{n, j}}{\left|w_{n, j}\right|} \frac{z-w_{n, j}}{1-\bar{w}_{n, j} z}, \quad b_{\mu}(z)=\prod_{n=1}^{\infty} b_{K_{n}}^{\prime}(z), \quad z \in D .
$$

Then $b_{\mu}$ is an interpolating Blaschke product and

$$
Z\left(b_{K_{n}}^{\prime}\right)=Z\left(b_{K_{n}}\right), \quad \bigcup_{n=1}^{\infty} Z\left(b_{K_{n}}\right) \subset Z\left(b_{\mu}\right) .
$$

Let $S$ be a measurable subset of supp $\mu$ such that $x \in \widehat{S}$. Since $\mu_{\mid S} \neq 0$, by (6.9) there exists a positive integer $n$ such that $\mu_{\mid K_{n} \cap S} \neq 0$. By (6.7) and Theorem 6.1, $Z\left(b_{K_{n}}\right) \cap \mathcal{Z}\left(\mu_{\mid K_{n} \cap S}\right) \neq \emptyset$. Then by $(6.10), Z\left(b_{\mu}\right) \cap \mathcal{Z}\left(\mu_{\mid K_{n} \cap S}\right)$ $\neq \emptyset$. Hence by Lemma 3.3 , we have $Z\left(b_{\mu}\right) \cap \mathcal{Z}\left(\mu_{\mid S}\right) \neq \emptyset$. In the same way 
as in the proof of Theorem $6.3(\mathrm{i})$, we have $Z\left(b_{\mu}\right) \cap \Phi_{\mu}(x) \neq \emptyset$. Since $b_{\mu}$ is interpolating, $\Phi_{\mu}(x)$ contains non-trivial points.

Problem 6.7. Let $\mu \in M_{\mathrm{s}}^{+}$and $x \in M\left(L^{\infty}(\mu)\right)$. Does $\Phi_{\mu}(x)$ contain sparse points?

The author would like to thank the referee for his/her comments on the first version of the manuscript.

\section{References}

[1] P. Budde, Support sets and Gleason parts, Michigan Math. J. 37 (1990), 367-383.

[2] L. Carleson, An interpolation problem for bounded analytic functions, Amer. J. Math. 80 (1958), 921-930.

[3] - Interpolations by bounded analytic functions and the corona problem, Ann. of Math. 76 (1962), 547-559.

[4] T. Gamelin, Uniform Algebras, Prentice-Hall, Englewood Cliffs, NJ, 1969.

[5] J. Garnett, Bounded Analytic Functions, Academic Press, New York, 1981.

[6] P. Gorkin, Decompositions of the maximal ideal space of $L^{\infty}$, Trans. Amer. Math. Soc. 282 (1984), 33-44.

[7] P. Gorkin, H.-M. Lingenberg, and R. Mortini, Homeomorphic disks in the spectrum of $H^{\infty}$, Indiana Univ. Math. J. 39 (1990), 961-983.

[8] C. Guillory and K. Izuchi, Interpolating Blaschke products of type G, Complex Variables 31 (1996), 51-64.

[9] H. Hedenmalm, Thin interpolating Blaschke products and three algebras of analytic functions, Proc. Amer. Math. Soc. 99 (1987), 489-495.

[10] K. Hoffman, Banach Spaces of Analytic Functions, Prentice-Hall, Englewood Cliffs, NJ, 1962.

[11] —, Bounded analytic functions and Gleason parts, Ann. of Math. (2) 86 (1967), 74-111.

[12] K. Izuchi, Weak infinite powers of Blaschke products, J. Anal. Math. 75 (1998), $135-154$.

[13] - Singular inner functions of $L^{1}$-type II, J. Math. Soc. Japan 53 (2001), 285-305.

[14] K. Izuchi and N. Niwa, Singular inner functions of $L^{1}$-type, J. Korean Math. Soc. 36 (1999), 787-811.

Department of Mathematics

Niigata University

Niigata 950-2181, Japan

E-mail: izuchi@math.sc.niigata-u.ac.jp

Received February 14, 2003

Revised version December 15, 2003 